EUROPEAN JOURNAL OF PURE AND APPLIED MATHEMATICS

Vol. 12, No. 2, 2019, 370-408

ISSN 1307-5543 - www.ejpam.com

Published by New York Business Global

\title{
Literature Survey on Non-associative Rings and Developments
}

Tariq Shah ${ }^{1}$, Asima Razzaque ${ }^{2, *}$ Inayatur Rehman ${ }^{3}$, Muhammad Asif Gondal ${ }^{3}$, Muhammad Iftikhar Faraz ${ }^{4}$, Kar Ping Shum ${ }^{5}$

1 Department of Mathematics, Quaid-e-Azam University, Islamabad, Pakistan

2 Department of Mathematics, University of Education, Lahore, Pakistan

3 Department of Mathematics and Sciences, Dhofar University, Salalah, Oman

4 College of Engineering, Grand Valley State University, USA.

${ }^{5}$ Institute of Mathematics, Yunnan University, Kunming, 650091, P. R. China.

\begin{abstract}
In this paper we present a comprehensive survey and developments of existing literature of non-associative rings and enumerate some of their various applications in different directions to date. These applications explain the voluminous work in different fields of non-associative rings and through which various algebraic structures in theoretical point of view could be developed.
\end{abstract}

2010 Mathematics Subject Classifications: 17D99

Key Words and Phrases: Octonions, Jordan Rings, LA-rings

\section{Introduction}

One of the endlessly alluring aspects of mathematics is that its thorniest paradoxes have a way of blooming into beautiful theories. Pure mathematics is, in its way the poetry of logical ideas. Today mathematics especially pure mathematics is not the same as it was hundred years ago. Many revolutions have occurred and it has taken new shapes with the due course of time. Until recently the theory of rings and algebras was regarded exclusively as the theory of associative rings and algebras. This was a result of the fact that the first rings encountered in the course of the development of mathematics were associative (and commutative) rings of numbers and rings of functions, and also associative rings of endomorphisms of abelian groups, in particular, rings of linear transformations of vector spaces. This survey of one part of the theory of rings: precisely, the theory of rings, which although non-associative, are more or less connected with the theory of associative rings. More precise connections will be mentioned during the discussion of particular classes of rings.

* Corresponding author.

DOI: https://doi.org/10.29020/nybg.ejpam.v12i2.3408

Email addresses: asima.razzaque@yahoo.com (A. Razzaque), stariqshah@gmail.com (T. Shah), irehman@du.edu.om (I. Rehman), mgondal@du.edu.om (M. A. Gondal), farazm@gvsu.edu (M. I. Faraz), kpshum@ynu.edu.cn (K. P. Shum) 
A major change took place in the mid of $19^{\text {th }}$ century when the concept of non-associative rings and non-associative algebras were introduced. The theory of non-associative rings and algebras has evolved into an independent branch of algebra, exhibiting many points of contact with other fields of mathematics and also with physics, mechanics, biology, and other sciences. The central part of the theory is the theory of what are known as nearly-associative rings and algebras: Lie, alternative, Jordan, loop rings and algebras, and some of their generalizations.

We briefly describe the origins of the theory of non-associative rings. The oldest nonassociative operation used by mankind was plain subtraction of natural numbers. The first ever example of a ring that is non-associative is Octonions, constructed by John T. Graves in 1843. On the other hand the first example of an abstract non-associative system was Cayley numbers, constructed by Arthur Cayley in 1845. Later they were generalized by Dickson to what we know as Cayley-Dickson algebras. Later in 1870 a very important non-associative class known as Lie Theory was introduced by the Norwegian mathematician Sophus Lie. He employed a novel approach, combining transformations that preserve a type of geometric structure (specifically, a contact structure) and group theory to arrive at a theory of continuous transformation groups [189]. Since then, Lie Theory has been found to have many applications in different areas of mathematics, including the study of special functions, differential and algebraic geometry, number theory, group and ring theory, and topology [99, 103, 109]. It has also become instrumental in parts of physics, for some Lie algebras arise naturally from symmetries in physical systems, and is a powerful tool in such areas as quantum and classical and mechanics, , solid state physics, atomic spectroscopy and elementary particles [34, 99, 109]. No doubt Lie theory is a fundamental part of mathematics. The areas it touches contain classical, differential, and algebraic geometry, topology, ordinary and partial differential equations, complex analysis and etc. And it is also an essential chapter of contemporary mathematics. A development of it is the Uniformization Theorem for Riemann surface. The final proof of such theorem is the invention from Einstein to the special theory of relativity and the Lorentz transformation. The application of Lie theory is astonishing. Moreover, in 1890's the concept of hyperbolic quaternion was given by Alexander Macfarlane which forms a non-associative ring that suggested the mathematical footing for space time theory that followed later.

Furthermore, to the best of our knowledge the first detailed discussion about Alternative rings was started in 1930 by the German author Zorn [268-271]. For more study about this nonassociative structure we refer the readers to study [2, 42, 110, 205-207]. Another important class of non-associative structures was introduced in 1932-1933 by German specialist Pasqual Jordan in his algebraic formulation of quantum mechanics. Jordan structures also appear in quantum group theory, and exceptional Jordan algebras play an important role in recent fundamental physical theories, namely, in the theory of super-strings [107]. The systematic study of general Jordan algebras was started by Albert in 1946. In addition, the study of loops started in 1920's and these were introduced formally first time in 1930's [200]. The theory of loops has its roots in geometry, algebra and combinatorics. This can be found in nonassociative products in algebra, in combinatorics it is presented in latin squares of particular form and in geometry it has connection with the analysis of web structures [199]. A detailed study of theory of the loops can be found in [3, 4, 21-23, 199]. Historically, the concept of 
a non-associative loop ring was introduced in a paper by Bruck in 1944 [20]. Non-associative loop rings appear to have been little more than a curiosity until the 1980s when the author found a class of non-associative Moufang loops whose loop rings satisfy the alternative laws.

After the concept of loop rings (1944), a new class of non-associative ring theory was given by Yusuf in 2006 [265]. Although the concept of LA-ring was given in 2006, but the systematic study and further developments was started in 2010 by Shah and Rehman in their paper [215]. It is worth mentioning that this new class of non-associative rings named Left almost rings (LA-ring) is introduced after a huge gap of 6 decades since the introduction of loop rings. Left almost rings (LA-ring) is actually an off shoot of LA-semigroup and LA-group. It is a noncommutative and non-associative structure and gradually due to its peculiar characteristics it has been emerging as useful non-associative class which intuitively would have reasonable contribution to enhance non-associative ring theory. By an LA-ring, we mean a non-empty set $R$ with at least two elements such that $(R,+)$ is an LA-group, $(R,$.$) is an LA-semigroup, both$ left and right distributive laws hold. In [215], the authors have discussed LA-ring of finitely nonzero functions which is in fact a generalization of a commutative semigroup ring. On the way the first ever definition of LA-module over an LA-ring was given by Shah and Rehman in the same paper [215]. Moreover, Shah and Rehman [216] discussed some properties of LArings through their ideals and intuitively ideal theory would be a gate way for investigating the application of fuzzy sets, intuitionistics fuzzy sets and soft sets in LA-rings. For example, Shah et al., [248] have applied the concept of intuitionistic fuzzy sets and established some useful results. In [106] some computational work through Mace4 has been done and some interesting characteristics of LA-rings have been explored. Further Shah et al., [247] have promoted the concept of LA-module and established some results of isomorphism theorems and direct sum of LA-modules. Recently, in 2014, Alghamdi and Sahraoui [8] have defined and constructed a tensor product of LA-modules and they extended some simple results from the ordinary tensor to the new setting. In 2014, Yiarayong [263] have given the new concept of left primary and weakly left primary ideals in LA-rings. Some characterizations of left primary and weakly left primary ideals are obtained. Moreover, in 2015 Hussain and Khan [104] have characterized LA-rings by congruence relations. They proved that each homomorphism of left almost rings defines a congruence relation on left almost rings. For some more study of LA-rings, we refer the readers to see $[202,213,217,246]$.

\section{Historical Perspective and Developments}

It is impossible in a short space to convey the full compass of the subject, but we will site some literature on non-associative rings from different decades. Here we tried to give the literature survey of all non-associative rings and their developments in different time periods including LA-rings (a class of non-associative rings), recently introduced in 2006.

\subsection{Octonions}

In order to solidify the non-associative ring theory, the origin of the non-associative ring could be traced to the work of John T. Graves who discovered Octonions in 1843, which is 
considered to be the first ever example of non-associative ring. It is an 8-dimensional algebra over $R$ which is non-associative as well as being non-commutative. These were rediscovered by cayley in 1845 and are also known sometimes as the cayley numbers. Each nonzero element of octonion still has an inverse so that it is a division ring, albeit a non-associative one. For a most comprehensive account of the octonions see [9]. The process of going from $R$ to $C$, from $C$ to $H$, and from $H$ to $O$, is in each case a kind of doubling process. At each stage something is lost from $R$ to $C$ it loosed the property that $R$ is ordered, from $C$ to $H$ loosed commutativity and from $H$ to $O$ loosed associativity. This process has been generalized to algebras over fields and indeed over rings. It is called Dickson doubling or Cayley-Dickson Doubling see [33, 198]. If we apply the Cayley-Dickson doubling process to the octonions we obtain a structure called the sedenions, which is a 16-dimensional non-associative algebra. In physics community much work is currently focused on octonion models see $[39,74,190,255]$. Historically speaking, the inventors or discoverers of the quaternions, octonions and related algebras (Hamilton, Cayley, Graves, Grassmann, Jordan, Clifford and others) were working from a physical point-of-view and wanted their abstractions to be helpful in solving natural problems [105].

\subsection{Lie Rings (1870-2015)}

In 1870 a very important non-associative class known as Lie Theory was introduced by the Norwegian mathematician Sophus Lie. The theory of Lie algebras is an area of mathematics in which we can see a harmonious between the methods of classical analysis and modern algebra. This theory, a direct outgrowth of a central problem in the calculus, has today become a synthesis of many separate disciplines, each of which has left its own mark. The importance of Lie algebras for applied mathematics and for applied physics has also become increasingly evident in recent years. In applied mathematics, Lie theory remains a powerful tool for studying differential equations, special functions and perturbation theory. Lie theory finds applications not only in elementary particle physics and nuclear physics, but also in such diverse fields as continuum mechanics, solid-state physics, cosmology and control theory. Lie algebra is also used by electrical engineers, mainly in the mobile robot control. For the basic information of Lie algebras, the readers are referred to [10, 31, 102].

It is well known that Lie algebra can be viewed as a Lie ring. So, the theory of Lie ring can be used in the theory of Lie algebra. A Lie ring is defined as a non-associative ring with multiplication that is anti-commutative and satisfies the Jacobi identity i.e. $[a,[b, c]]+$ $[b,[c, a]]+[c,[a, b]]=0$

Although the Lie theory was introduced in 1870 but the major developments were made in the 20th century with the paper of Hausdorff [81] in 1906. In (1934-35), Ado [1] proved that any finite dimensional Lie algebra over the field of complex numbers can be represented in a finite dimensional associative algebra. Moreover, in 1937, Birkhoff [12] and Witt [260] independently examined that every Lie algebra is isomorphic to sub-algebra of some algebra of the form $A^{(-)}$, where $A^{(-)}$is a Lie ring defined by $x . y=x y-y x$. They also found a formula for computing the rank of the homogeneous modules in a free Lie algebra on a finite number of generators. Also in 1937, Magnus [166] proved that the elements $y i=1+x i$ of the ring $H$ generate a free subgroup $G$ of the multiplicative group of the ring $H$, and every element of 
the subgroup $G_{n}$ (the $n$-th commutator subgroup) has the form $1+\ln +w$, where $\ln$ is some homogeneous Lie polynomial (with respect to the operations $x . y$ and $x+y$ of degree $n$ in the generators $a i$, and $w$ is a formal power series in which all the terms have degree greater than $n$.

In 1947, Dynkin [43] gave the criteria to determine whether the given polynomial is a Lie polynomial. Later in (1948-49), Harish Chandra [78] and Iwasawa [108] proved that Ado's theorem holds for any finite dimensional Lie algebra. Moreover, an important role in the theory of Lie rings is played by free Lie rings. In contrast to free alternative rings and free $J$-rings (free Jordan-rings), free Lie rings have been thoroughly studied. In that context, in 1950, Hall [75] pointed out a method for constructing a basis of a free Lie algebra. In addition, analogous theorems about embedding of arbitrary algebras and of associative rings were proved respectively by Zhukov [267] in 1950 and by Malcev [174] in 1952.

In (1953-54), Lazard [158] and Witt [261] studied representations of $\sum$-operator Lie rings in $\sum$-operator associative rings. The existence of such a representation was proved by them in the case of $\sum$-principal ideal rings and in particular for Lie rings without operators. The example constructed by Shirshov in [219] shows that there exist non-representable $\sum$-operator Lie rings which do not have elements of finite order in the additive group. Also in 1954, Higgins [94] investigated that solvable rings satisfying the $n$-th Engel condition are nilpotent and in continuation, Lazard [159] studied nilpotent groups using large parts of the apparatus of Lie ring theory.

In 1955, Cohn [32] constructed an example of a solvable Lie ring, with additive $p$-group (in characteristic $p$ ) and satisfying the $p$-th Engel condition, which is not nilpotent. Lie rings with a finite number of generators and some restrictions on the additive group. Also in 1955, Malcev [175] considered a class of binary-Lie rings, which are related to lie rings in a way analogous to the way alternative rings are related to associative rings. In (1955-56), Herstein [85-87] discussed associative rings which are dedicated to studying the rings $A^{(-)}$with different assumptions on the ring $A$. In 1956, Witt [262] proved that any sub-algebra of a free Lie algebra is again free. This theorem is analogous to the theorem of Kurosh for sub-algebras of free algebras. In the year 1957, many authors work on Lie algebra. For example, Higman [96] proved nilpotency of any Lie ring which has an automorphism of prime order without nonzero fixed points. This statement allowed him to prove nilpotency of finite solvable groups which have an automorphism satisfying the analogous condition. Gainov [52], investigated that in the case of a ring for which the additive group has no elements of order two, for a ring to be binary-Lie it is sufficient that these identities hold: $a^{2}=[(a b) b] a+[(b a) a] b=0$. The author proposed that on the set of elements of some alternative ring $D$, we can define the above described operation $x . y=x y-y x$ and then it implies that in the ring $D^{(-)}$, these relations hold identically: $a^{2}=[(a \cdot b) \cdot c] \cdot a+[(b \cdot c) \cdot a] \cdot a+[(c \cdot a) \cdot a] \cdot b-(a \cdot b) \cdot(a \cdot c)=0$. Rings satisfying these identities are called Moufang-Lie rings. He showed that the class of Moufang-Lie rings without elements of additive order 6 is properly contained in the class of binary-Lie rings. In (1957-58), Kostrikin [147] proved that the Engel condition implies nilpotency. This result is especially interesting because from it follows the positive solution of the group-theoretical restricted Burnside problem for $p$-groups with elements of prime order [145, 146].

Herstein and Kleinfeld [93] in 1960, discussed that the mappings $\phi$ onto a simple ring 
of characteristic 2 which preserve commutators and cubes. This situation is of interest for in characteristic 2 Jordan homomorphisms are the same thing as Lie homomorphisms, that is, mappings which preserve commutators. In 1961, Macdonald [164] established analogous results that are valid for some varieties of Lie rings. It is natural to bring some methods in finite group theory into the study of Lie algebras. Herstein [89] in 1961 gave us the idea of Lie and Jordan structures in simple associative rings. In 1963, Kreknin [148] examined that if a Lie ring $L$ admits a regular automorphism $\phi$ of finite order $k$, that is, such that $\phi k=1$ and $C L(\phi)=0$, then $L$ is soluble of derived length bounded by a function of $k$, actually, by $2 k-2$. $\mathrm{He}$ also discussed the bounded solubility of a Lie ring with a fixed-point-free automorphism, but the existing Lie ring methods cannot be used for bounding the derived length in general. Moreover, Kreknin and Kostrikin [150] in 1963 suggested that a Lie ring with a fixed-point-free automorphism of prime order $p$ is nilpotent of $p$-bounded class. In continuation Kreknin and Kostrikin also investigated that a Lie ring (algebra) admitting a regular (i.e., without nontrivial fixed points) automorphism of prime order $p$ is nilpotent of class bounded by a function $h(p)$ depending only on $p$. Kreknin [149] in 1967 projected that a Lie ring (algebra) admitting a regular automorphism of finite order $n$ is soluble of derived length bounded by a function of $n$. In 1969, Herstein [91] focused his study on the structures of the Jordan and Lie rings of simple associative rings. In the latter case the approach is via the study of the structure of $I(R)$, the Lie ring of inner derivations of $R$, or, equivalently, the Lie structure of $R / Z$.

In 1970, Herstein [92] studied lie structure of associative rings and proved some important results regarding lie structure of $R / Z$. In 1972, Lanski and Montgomery [157] studied Lie structure of prime rings of characteristic 2 . Results on Lie ideals were obtained. These results were then applied to the group of units of the ring, and also to Lie ideals of the symmetric elements when the ring has an involution. This work extends recent results of Herstein, Lanski and Erickson on prime rings whose characteristic is not 2, and results of $\mathrm{S}$. Montgomery on simple rings of characteristic 2 . In 1974, Kawamoto [128] discussed prime and semiprime ideals of Lie rings and showed that in a Lie algebra satisfying the maximal condition for ideals, any semi-prime ideal is an intersection of finite number of prime ideals and the unique maximal solvable ideal is equal to the intersection of all prime ideals. Jordan et al.[120] in 1978, studied that how the ideal structure of the Lie ring of derivations of $R$, is determined by the ideal structure of $R$. Moreover, the authors were interested in extending these results to the case where $R$ is a prime or semi-prime ring. Hartley et al., [79], in 1981 and Khukhro [129] in 1986 proposed that the results on Lie rings with regular or almost regular automorphisms of prime order have consequences for nilpotent (or even finite, or residually locally nilpotent-by-finite, etc.) groups with such automorphisms.

In 1992, Khukhro [130] has generalized the work of Kreknin and Kostrikin [148, 150] on regular automorphisms; (almost) regularity of an automorphism of prime order implied (almost) nilpotency of the Lie ring (algebra), with corresponding bounds for the nilpotency class and the index (co-dimension). He also showed that a Lie ring (algebra) $L$ admitting an automorphism $\phi$ of prime order $p$ with finite fixed-point sub-ring of order $m$ (with finite-dimensional fixedpoint sub-algebra of dimension $m$ ) has a nilpotent sub-ring (sub-algebra) $K$ of class bounded by a function of $p$ with the index of the additive subgroup $|L: K|$ (the co-dimension of $K$ ) bounded by a function of $m$ and $p$. Moreover, Khukhro proved that if a periodic (locally) 
nilpotent group $G$ admits an automorphism $\phi$ of prime order $p$ with $m=|C G(\phi)|$ fixed points then $G$ has a nilpotent subgroup of $(m, p)$ - bounded index and of $p$-bounded class and on the way this group result was also based on a similar theorem on Lie rings. The result given in [130], was later extended by Medvedev [180] in 1994 to not necessarily periodic locally nilpotent groups. In 1996 and in 1998, the authors [169-171] developed a method of graded centralizers given in [130] to study the almost fixed-point-free automorphisms of Lie rings and nilpotent groups. Medvedev [181] in 1999 Zapirain [266] in 2000 and Makarenko [167] in 2001 established the most successful case regarding the nilpotent (or finite) $p$-groups with an almost regular automorphism of order $p_{n}$, where theorems on regular automorphisms of Lie rings were used.

Great progress has been made to date in Lie rings (algebras) with almost regular automorphisms. The history of this area of research started with the classical theorem of Kreknin. In 2003, Khukhro and Makarenko [131] proved that if a Lie ring admits an automorphism of prime-power order that is almost regular then $L$ is almost soluble. Moreover, in 2003 and in 2004 Makarenko and Khukhro [172, 173], have succeeded in investigating the most general case of a Lie ring (algebra) with almost regular automorphism of arbitrary finite order. Makarenko and Khukhro [173] in 2004 analyzed that almost solubility of Lie rings and algebras admitting an almost regular automorphism of finite order, with bounds for the derived length and co-dimension of a soluble sub-algebra, but for groups even the fixed-point-free case remains open. In 2005, Kuzucuoglu [153] proved isomorphisms between finitary unitriangular groups and those of associated Lie rings are studied. The author also investigated its exceptional cases. Makarenko [168] in 2005, improved the conclusion in Khukhro's theorem stating that a Lie ring (algebra) $L$ admitting an automorphism of prime order $p$ with finitely many $m$ fixed points (with finite-dimensional fixed-point sub-algebra of dimension $m$ ) has a sub-ring (sub-algebra) $H$ of nilpotency class bounded by a function of $p$ such that the index of the additive subgroup $|L: H|$ (the co-dimension of $H$ ) is bounded by a function of $m$ and $p$. He proved that there exists an ideal, rather than merely a sub-ring (sub-algebra), of nilpotency class bounded in terms of $p$ and of index (co-dimension) bounded in terms of $m$ and $p$.

In 2008, Suanmali [243] used an analogous idea in the theory of group varieties to investigate the varieties of Lie algebras. She considered the exponent bound problem for some varieties of nilpotent Lie algebras and extended [164, 165] Macdonald's results to finite-dimensional Lie algebras over a field of characteristic not 2 and 3. Paul and Sabur Uddin [194] in 2010 worked on Lie and Jordan structure in simple gamma rings. They obtained some remarkable results concerning to Lie and Jordan structure. In 2010, Paul and Sabur Uddin [195] focused their discussion to the study Lie structure in simple gamma rings. They gave us some structural results of simple gamma rings with Lie ideals.

In 2011, Khukhro, Makarenko and Shumyatsky [45] developed a Lie ring theory which is used for studying groups $G$ and Lie rings $L$ with a metacyclic Frobenius group of automorphisms $F H$. Wilson [258] in 2013 introduced three families of characteristic subgroups that refined the traditional verbal subgroup filters, such as the lower central series, to an arbitrary length. It was proved that a positive logarithmic proportion of finite $p$-groups admit at least five such proper nontrivial characteristic subgroups whereas verbal and marginal methods explained only one. The placement of these subgroups in the lattice of subgroups is naturally recorded by 
a filter over an arbitrary commutative monoid $M$ and induces an $M$-graded Lie ring. These Lie rings permit an efficient specialization of the nilpotent quotient algorithm to construct automorphisms and decide isomorphism of finite $p$-groups. In 2013, Khukhro and Makarenko [132] discovered that the representation theory arguments are used to bound the index of the fitting subgroup. Lie ring methods are used for nilpotent groups. A similar theorem on Lie rings with a metacyclic Frobenius group FH of automorphisms was also proved. In 2014, Horn and Zandi [98] the aim in their paper is to gave an explicit description of the cohomology group $H^{2}(L, A)$ and to show how its elements correspond one-to-one to the equivalence classes of central extensions of the Lie algebra $L$ with the module $A$, where we regard $A$ as abelian Lie ring. More recently in 2015, Wilson [259] generalized the common notion of descending and ascending central series. The descending approach determines a naturally graded Lie ring and the ascending version determines a graded module for this ring. He link derivations of these rings to the automorphisms of a group.

\subsection{Alternative Rings (1930-2015)}

To the best of our knowledge the first detailed discussion about alternative rings was started in 1930 by the German author Zorn. An alternative ring $R$ is defined by the system of identities: $(a b) b=a(b b)$ (right alternativeness) and $(a a) b=a(a b)$ (left alternativeness) for all $a, b \in R$.

In 1930, Zorn [268] mentioned the theorem of Artin which states that every two elements of an alternative ring generate an associative sub-ring. By a result of Zorn [268], it was observed that the only not associative summands permitted are merely finite Cayley-Dickson algebras (which is the first example of alternative rings) with divisors of zero. In 1933, Zorn [269] discussed also the finite-dimensional case in alternative rings. In 1935, Moufang [186] proved a generalization for alternative division rings: if $(a, b, c)=0$, then $a, b, c$ generate a division sub-ring which is associative. For more details regarding finite dimensional case the readers are referred to the contribution of Jacobson [110], Albert [2], Schafer [206, 207] Dubisch and Perlis [42]. In 1943, Schafer [205] studied the alternative division algebras of degree two which is independent of Zorn's results. In 1946, Forsythe and McCoy [51] gave an approach that an associative regular ring without nonzero nilpotent elements is a sub-direct sum of associative division rings is easily extendable to alternative rings. In 1947, Smiley [237] studied alternative regular rings without nilpotent elements and proposed an approach that every alternative algebraic algebra which has no nilpotent elements is the sub-direct sum of alternative division algebras. Kaplansky [126] in 1947 presented many of the preliminary results which were valid at least for special alternative rings. Smiley [238] in 1948 studied the concept of radical of an alternative ring and discussed the radicals of infinite order algebras and was also able to show that the Jacobson's definition of the radical of an associative ring is applied to alternative ring. In 1948, Kaplansky [125] also obtained the Cayley numbers as the only not associative alternative division ring which was both connected and locally connected, and he gave a conjecture that a similar result holds in the totally disconnected, locally compact case. A ring is defined to be right alternative in case $a b . b-a . b b=0$ is an identical relation in the ring. Right alternative algebras were first studied by Albert [6] in 1949 he showed that a 
semi-simple, right alternative algebra over a field of characteristic 0 is alternative.

In 1950, Brown and McCoy [17] suggested that every alternative ring has a greatest regular ideal. Also in 1950, in the work of Skornyakov [221, 222] provided a full description of alternative but not associative division rings. He showed that each such division ring is an algebra of dimension 8 over some field. Later in 1951, Bruck and Kleinfeld [24] proved the result of Skornyakov [221], independently. In 1951, Skornyakov [223] proposed that the study of alternative rings in general began with the study of alternative division rings, which in the theory of projective planes play the role of the so-called natural division rings of alternative. Another result concerns right alternative division rings, which are of geometrical interest since they arise as coordinate systems of certain projective planes in which a configuration weaker than desargue's is assumed to hold. In this connection Skorniakov [225] in 1951 has made known that a right alternative division ring of characteristic not 2 is alternative. Some attention had been given to right alternative rings when Skornyakov [224] in 1951 established the result that every right alternative division ring is alternative.

In 1952, Albert [7] proved the results for simple alternative rings and his proposed results were based on the properties which were given by Zorn [268]. Kleinfeld [134] in 1953 proved that for the alternativity of a right alternative ring it is sufficient that $[x, y, z]^{2}=0$ implies $[x, y, z]=0$. Kleinfeld [135] in 1953 proved that even simplicity (that is, not having two-sided ideals) of an alternative but not associative ring implies that the ring is a Cayley-Dickson algebra. In 1953, Kleineelo [133] proved that right alternative rings without nilpotent elements are known to be alternative it follows that free right alternative rings with two or more generators have non-zero nilpotent elements. In 1955, Kleinfeld [136] strengthened his results by proving that any alternative but not associative ring, in which the intersection of all the two-sided ideals is not a nil ideal, is Cayley-Dickson algebra over some field. Hence the class of alternative rings is much larger than the class of associative rings. San Soucie [241, 242] in 1955 studied alternative and right alternative rings in characteristic $2(2 x=0)$ and also proved that if $R$ is right alternative division ring of characteristic two. Then $R$ is alternative if and only if $R$ satisfies $w(x y-x)=(w x-y) x$. In 1957, Kleinfeld [240] proved very interesting identity: $\left[(a b-b a)^{2}, c, d\right](a b-b a)=0$ and he also showed that in the free alternative ring there are zero divisors. Smiley [239] in 1957 analyzed the proof of Kleinfeld and noticed that it is sufficient to check only these cases: $x=y, x=y z-z y, x=(y z-z y) y, x=[y, y, z]$, or $z=w y$ and $x=[y, y, w]$ for some $w$. To study of free right alternative rings he said that it was one of the main tasks of the theory of alternative rings.

In 1960, Hashimoto [80] introduced the notion of $*$ - modularity of right ideals of an alternative rings and showed a connection between the intersection of all the $*$ - modular maximal right ideals and the radical $S R(A)$ in an alternative ring $A$. In 1963, it was shown by Kleinfeld [137] that in an arbitrary alternative ring the fourth power of every commutator lies in the nucleus. Also Dorofeev [41] in 1963 proved that in a free alternative ring with six or more generators there exist elements $a, b, c, d, r, s$ such that $((a, b)(c, d)+(c, d)(a, b), r, s) \neq 0$. In 1965, Slater [226] asserted that a prime alternative ring $R$ of characteristic not 3 that is not associative can be embedded in a Cayley-Dickson algebra over the quotient field of the center of $R$. In 1967, Humm [100] discussed a necessary and sufficient condition for a simple right alternative ring to be alternative. He assumed that the characteristic is not 2 or 3 in all that 
follows. The treatment required an idempotent $e$ in $R$ and used the subspaces $R_{1}(e)$ and $R_{0}(e)$ of the Albert decomposition [5]. In 1967, Humm and Kleinfeld [101] investigated that with the help of an example that square of every commutator need always lie in the nucleus. Also, they showed the existence of specific nilpotent elements in the free alternative ring on four or more generators, and proved abstractly the existence of an ideal $I \neq 0$, and $I^{2}=0$. Slater [227] in 1967 in his paper on nucleus and center in Alternative rings considered $R$ is any alternative ring, $N$ its Nucleus and $Z$ its center. Moreover, he investigated the natural conditions on $R$ which were the weakest possible to ensure. Also applied the results to amplify comments by Humm and Kleinfeld work on free alternative rings and contained examples of alternative rings. Slater [228] in 1968 discussed the ideals in semiprime alternative rings and also the results of the paper, so far concerned that a given right ideal $A$, did not require semiprimeness of $R$. In 1969, Kleinfeld [139] worked on right alternative rings without proper right ideals he showed that a right alternative ring $R$ without proper right ideals, of characteristic not two, containing idempotents $e$ and $1, e \neq 1$, such that $e x=e(e x)$ for all $x \in R$ must be alternative and hence a cayley vector matrix algebra of dimension 8 over its center. Moreover, Slater [229] in 1969, proved the natural extension to arbitrary rings of the classical Wedderburn-Artin theorem for associative ones. Also considered the special case where $R$ is in addition purely alternative; that is, has no nonzero nuclear ideals. He also listed virtually all the radicals that have been proposed for (alternative) rings in the literature, and showed that on the class of rings with D.C.C. they all coincide. Also he discussed analogous for arbitrary rings with D.C.C. of the classical results concerning idempotents in associative rings with D.C.C.

In 1970, Slater [231] discussed the class of admissible models. Since a prime ring need not be algebra over a field, so keeping in view, the author intended to extend the class of admissible models at least slightly. For example, the Cayley integers are a prime ring that is not Cayley-Dickson algebra, much as an integral domain is prime but need not be a field. Moreover, he defined a Cayley-Dickson ring (CD ring) $R$ to be a ring that can be imbedded in a certain natural way in CD algebra $R$ over the quotient field $Z$ of the (nonzero) center $Z$ of $R$. He then later said that if $R$ is cancellative alternative but not associative (and of char $\neq 2$ ) then $R$ is a CD ring such that $R$ is a CD division algebra. The added generality in the paper comes from the fact that a prime ring may have zero divisors. If $R$ is prime with zero divisors [and not associative, and $3 R \neq(0)$ ] then $R$ will be a split CD algebra, instead of a CD division algebra. Again in 1970, Slater [232] discussed localization results on ideals and right ideals of prime and weakly prime rings. Also he showed that if some exceptional weakly prime ring exists, then there exists an exceptional prime ring having a collection of properties which taken together. Finally, he gave examples to show that if some exceptional ring exists, then the restrictions on characteristic imposed in most of the results were not excessive. Slater [230] in 1970 proved the natural extension to alternative rings of the classical Wedderburn-Artin theorem for semiprime associative rings, considered the extension to arbitrary alternative rings of the classical methods, as well as the secondary results of the classical associative theory. Also he discussed some parallel conditions in alternative theory to the classical connection between primitive idempotents and minimal right ideals. He also examined the relation between the present results and the classical structure theory established by Zorn. In 1970, Slater [233] discussed that the main facts about the minimal ideals and minimal right ideals of an associative 
ring are well known. In this paper he also proved corresponding results for an alternative ring $R$. He made no restriction on the characteristic of $R$, but will often impose restrictions of semiprimeness type.

Slater [234] in 1971 were concerned mainly with the extension to arbitrary (alternative) rings of Hopkins theorem [97] that in an associative ring with D.C.C. on right ideals the (say, nil) radical is nilpotent. He also reworked and modified Zhevlakov's arguments to obtain nilpotence of $S(R)$ without restriction on characteristic. It turns out that much of the work was done more simply by working with two-sided ideals, as opposed to the right ideals used by Zhevlakov. As a consequence, a substantial part of the work was done with the assumption of D.C.C. only on two-sided ideals, and the result on $S(R)$ appeared as an easy corollary of this work. On the way he also improved the result that a ring $R$ with D.C.C. on two-sided ideals any solvable ideal is nilpotent by allowing Baer-radical ideals in place of solvable ideals. In 1971, Hentzel [82] discussed the characteristics of right alternative rings with idempotents, he also assumed that all the rings to have characteristic prime to 2 and 3 . In his paper he also used the Albert decomposition for idempotents for right alternative rings. In 1971, Kleinfeld [141] discussed that alternative as well as Lie rings satisfy all of the following four identities : $(i)\left(x^{2}, y, z\right)=x(x, y, z)+(x, y, z) x,(i i)\left(x, y^{2}, z\right)=y(x, y, z)+(x, y, z) y,(i i i)$ $\left(x, y, z^{2}\right)=z(x, y, z)+(x, y, z) z,(i v)(x, x, x)=0$, where the associator $(a, b, c)$ is defined by $(a, b, c)=(a b) c-a(b c)$. He also proved that if $R$ is a ring of characteristic different from two and satisfies (iv) and any two of the first three identities, then a necessary and sufficient condition for $R$ to be alternative is that whenever $a, b, c$ are contained in a sub-ring $S$ of $R$ which can be generated by two elements and whenever $(a, b, c)^{2}=0$, then $(a, b, c)=0$. Also all such division rings must be alternative and hence either Cayley-Dickson division algebras or associative. Also Kleinfeld [140] in 1971 investigated rings $R$ of characteristic different from two. The main results were concerned that either rings which have an idempotent $e \neq 1$, or those which have no nilpotent elements. He also proved that whenever $R$ is simple and contains an idempotent $e \neq 1$, then $R$ must be alternative and hence either a cayley vectormatrix algebra or associative.

In 1975, Thedy in his paper [250] analyzed the two natural concepts in a right alternative algebra $R$, the sub-module $M$ generated by all alternators $(x, x, y)$, and a new nucleus $N$. The later sections of his study dealt mainly with results on simple right alternative algebras. A simple 2-torsion free right alternative algebra is either alternative, hence associative or Cayley algebra over its center. Also in 1975, the work of Hentzel [83] was dealt with a GRA (generalized right alternative) ring $R$. It was shown that $I$ is an ideal of $R$, that $I$ is commutative, and that $I$ is the sum of ideals of $R$ whose cube is zero. This means that if $R$ is simple, or even nil-semisimple, and then $R$ is right alternative. Since all the hypotheses on $R$ are consequences of the right alternative law, showing that $R$ is right alternative is as strong a result. Also he considered that the ideal generated by each associator of the form $(a, b, b)$ is a nilpotent ideal of index at most three. Miheev [183] in 1975 constructed a finite-dimensional, prime, right alternative nil algebra with nilpotent heart. Thus a prime right alternative ring need not be $s$-prime. In 1976, Rich [203] discussed the characterization by Levitzkiin 1951 of the prime radical of an associative ring $R$ as the set of strongly nilpotent elements of $R$ was adapted to apply to a wide class of non-associative rings. As a consequence it was shown that the 
prime radical is a hereditary radical for the class of alternative rings and that the prime radical of an alternative ring coincides with the prime radical of its attached Jordan ring. In 1978, Rose [204] first gave the brief introduction of Cayley-Dickson algebra. He then axiomatized split Cayley-Dickson algebras over algebraically closed fields and showed that this theory is $\aleph_{1}$-categorical, model complete, and the model completion of the theory of Cayley-Dickson algebras and stability in alternative rings. He also generalized $\aleph_{0}$-categoricity in associative rings to $\aleph_{0}$-categoricity in alternative rings.

In 1980, Wene in his paper [256] characterized those associative rings with involutions in which each symmetric element is nilpotent or invertible. Analogous results were obtained for alternative rings. The restriction was further relaxed to require only that each symmetric element is nilpotent or some multiple is a symmetric idempotent. Widiger [257] in 1983 considered the class of all alternative rings in which every proper right ideal is maximal. Moreover, he used the theory of artinian rings for his study. Kleinfeld [142] in 1983 examined that a semiprime alternative ring can have no nonzero anti-commutative elements. However, this was not so for prime right alternative rings in general. In 1988, Essannouni and Kaidi [47] proved the natural extension to alternative rings of the classical Goldie theorem for semiprime associative rings.

In 1994, Essannounia and Kaidi [48] discovered that the socle of a semiprime Goldie ring is generated by a central idempotent and that a prime Goldie ring with a nonzero socle is a simple artinian ring. They also extended these results to alternative rings. They had given an analogue of Goldie's theorem for alternative rings. A Goldie like theorem was obtained earlier by the authors for noetherian alternative rings by a quite different method. Also in 1994, Kleinfeld and Smith [143] discussed that a ring is called $s$-prime if the 2-sided annihilator of a nonzero ideal must be zero. In particular, any simple ring or prime $(-1,1)$ ring is $s$-prime. Also, a nonzero $s$-prime right alternative ring, with characteristic $\neq 2$, cannot be right nilpotent.

In 2000, Goodaire [59] developed that for a right alternative ring $R$, the magma $(R, \circ)$ is right alternative, that is, $(x \circ y) \circ y=x \circ(y \circ y)$, and if $R$ is strongly right alternative, then $(R, \circ)$ is a Bol magma with neutral element 0. Moreover, in 2001, Goodaire [60] showed that in a strongly right alternative ring with unity, it was known that if $U(R)$ is closed under multiplication, then $U(R)$ is a Bol loop. Kenneth Kunen and Phillips [163] in 2005 partially answered two questions of Goodaire by showing that in a finite, strongly right alternative ring, the set of units (if the ring is with unity) is a Bol loop under ring multiplication, and the set of quasi-regular elements is a Bol loop under circle multiplication. Again in 2005, Cárdenas et.al., [154] studied the notion of a (general) left quotient ring of an alternative ring and showed the existence of a maximal left quotient ring for every alternative ring that is a left quotient ring of itself. In 2007, Lozano and Molina [162] developed a fountain Gould-like Goldie theory for alternative rings. They characterized alternative rings which were Fountain-Gould left orders in semiprime alternative rings coinciding with their socle, and those which were Fountain-Gould left orders in semiprime artinian alternative rings.

Furthermore, Bharathi et al., [35] in 2013 proved that if $R$ is a semiprime and purely non-associative right alternative ring, then $N=C$. They also showed that the right nucleus $N_{r}=C$ if $R$ is purely non-associative provided that either $R$ has no locally nilpotent ideals or $R$ is semi-prime and finitely generated $\bmod N_{r}$. In 2014, Cárdenas et al., [155] introduced 
a notion of left non-singularity for alternative rings and proved that an alternative ring is left non-singular if and only if every essential left ideal is dense, if and only if its maximal left quotient ring is von Neumann regular. Finally, they obtained a Gabriel-like Theorem for alternative rings. Ferreira and Nascimento [50] in 2014 proved the relationship between the multiplicative and the additive structures of a ring that became an interesting and active topic in ring theory. They focused their discussion on the special case of an alternative ring. In this they investigated the problem of when a derivable map must be an additive map for the class of alternative rings. Recently, in 2015, Satyanarayana et al., [264] proved that the peculiar property of nucleus $\mathrm{N}$ in an alternative ring $R$ i.e. nucleus contracts to centre $C$ when alternative ring is octonion and nucleus expands to whole algebra when the alternative ring is associative. Also in 2015, Jayalakshmi and Latha [117] presented some properties of the right nucleus in generalized right alternative rings. Also they showed that in a generalized right alternative ring $R$ which is finitely generated or free of locally nilpotent ideals, the right nucleus $N_{r}$ equals the center $C$. They also considered the ring to be generalized right alternative ring and tried to prove the results of $\mathrm{Ng}$ Seong-Nam [212]. On the way they gave an example to show that the generalized right alternative ring is not right alternative.

\subsection{Jordan Rings(1933-2011)}

In modern mathematics, an important notion is that of non-associative structure. This kind of structures is characterized by the fact the product of elements verifies a more general law than the associativity law. Jordan structures were introduced in 1932-1933 by the German physicist Pasqual Jordan (1902-1980) in his algebraic formulation of quantum mechanics. The study of Jordan structures and their applications is at present a wide-ranging field of mathematical research. The systematic study and more developments of general Jordan algebras were started by Albert in 1946. One can define a Jordan ring as a commutative non-associative ring that respects the Jordan identity i.e. $(x y)(x x)=x(y(x x))$.

In 1948, Jacobson [111] observed that semi-isomorphisms were nothing more or less than ordinary isomorphisms of the non-associative Jordan ring determined by the given associative ring. In his paper he introduced the Jordan multiplication $a . b=1 / 2(a b+b a)$, he observed that if ordinary multiplication is replaced by this identity then one can obtained Jordan ring determined by the associative ring. He also determined the isomorphisms between any two simple Jordan rings. Jacobson [112] in 1948 in his paper discussed about the centre of nonassociative ring i.e.; If $\Re$ is any non-associative ring one can defined the center of $\Re$ to be the totality of elements $c$ that commute, $c . a=$ a.c. It was also observed that if a ring contains a nilpotent element in its center then it contains a nilpotent two-sided ideal.

In 1950, Jacobson and Rickart [115] defined a special Jordan ring to be a subset of an associative ring which is a subgroup of the additive group and which is closed under the compositions $a \rightarrow a^{2}$ and $(a, b) \rightarrow a b a$. Such systems are also closed under the compositions $(a, b) \rightarrow a b+b a=\{a, b\}$ and $(a, b, c) \rightarrow a b c+c b a$. The simplest instances of special Jordan rings were the associative rings themselves. The authors also studied the (Jordan) homomorphism of these rings. Jacobson and Rickart [116] in 1952 considered the set $H$ of self- adjoint elements $h=h^{*}$. Then that set $H$ is a special Jordan ring. In this paper they 
studied the homomorphism of the rings of this type. They also obtained an analogue of the matrix method for the rings $H$. Authors proved that any Jordan homomorphism of $H$ can be extended to an associative homomorphism of $U$. They also examined that this result can be extended to locally matrix rings and in this form it is applicable to involutorial simple rings with minimal one-sided ideals. On the way they obtained the Jordan isomorphisms of the Jordan ring of self-adjoint elements of an involutorial primitive ring with minimal one-sided ideals onto a second Jordan ring of the same type. However, comparatively Schafer [208] in 1955 began the study of the class of so-called non-commutative $J$-rings (Jordan rings). The study of this class of rings is contained in the theory of algebras of finite dimension. For more details readers were referred to study $[144,209,210]$. In 1956, Hall and Jr [76] established the identity $\{a b a\}^{2}=\left\{a\left\{b a^{2} b\right\} a\right\}$ which hold in abstract Jordan rings. This was immediate for special Jordan rings. They examined that the identity is proved by finding a partial basis for the free Jordan ring with two generators, the basis being found for all elements of degree at most 5 and for elements of degree 4 in a and degree 2 in $b$. Herstein [88] in 1957 gave us the idea of derivation of Jordan ring. He mentioned that for any associative ring $A$, from its operations and elements a new ring can be obtained, that is the Jordan ring of $A$, by defining the product in the ring to be $a o b=a b+b a$ for all $a, b \in A$. In 1958, Shirshov [220] has made a detailed discussion of non-associative structures including Jordan rings. He also constructed some special Jordan rings.

In 1963, Brown [19] pointed out a problem of interest in non-associative algebras, regarding the study of generalized Cayley algebras and exceptional simple Jordan algebras which were closely related to the exceptional simple Lie algebras. In his work, he defined a new class of simple non-associative algebras of dimension 56 over their centers and possessing nondegenerate trace forms, such that the derivations and left multiplications of elements of trace zero generate Lie algebras of type $E_{7}$. Moreover, in 1964, Kleinfeld [138] gave the concept of middle nucleus and center in simple Jordan ring. He established the result that in a simple Jordan ring of characteristic $\neq 2$ the middle nucleus and center coincide. McCrimmon [176] in 1966 discussed about the structure, characteristics and general theory of Jordan rings. A Jordan ring (i.e., algebra over the ring of integers) is called non-degenerate if it has no proper absolute zero divisors. He also described that a Jacobson ring is a Jordan ring such that, the descending chain condition holds for Peirce quadratic ideals, and each nonzero Peirce quadratic ideal contains a minimal quadratic ideal. These rings play a role in the Jordan theory analogous to that played by the artinian rings in the associative theory. In 1968, Tsai [251] pointed up that there were several definitions of radicals for general non-associative rings given in literature. The $u$-prime radical of Brown-McCoy which was given in [18] was similar to the prime radical in an associative ring. However, it depends on the particular chosen element $u$. The purpose of the paper was to project a definition for the Brown-McCoy type prime radical for Jordan rings so that the radical will be independent from the element chosen. Tsai [252] in 1969 proved that in any Jordan ring $J$ there exists a maximal Von Neumann regular ideal $M$. The existence of such an ideal in an associative ring $A$ is a well known. In fact, $M$ could be characterized as the set of all elements $a$ in $A$ such that any element in the principal ideal in $A$ generated by $a$ is a regular element. He also had shown that the same characterization holds for Jordan rings. Also, in 1969, McCrimmon [177] established a self-contained proof which does not depend on 
the classification of simple rings. The author has taken motivation for this proof from the work of Jacobson [114] in which he has provided the proof in which he used the structure theory to reduce the problem to the case of simple rings, and then proceeded to check the result for each of the various types of simple rings that can occur.

Furthermore, in 1970, Meyberg [182] established a proof of Fundamental-Formula which is considered to be a very important in Jordan rings and given a comparatively short proof of Fundamental-Formula as first it was given by Jacobson [114]. Osborn [191] in 1970 presented three related theorems, one on the structure of Jordan rings in which every element is either nilpotent or invertible, and two on the structure of associative rings with involution in which every symmetric element is either nilpotent or invertible. The first of these theorems was a generalization of a well-known result on the structure of Jordan algebras which stated that if each element of Jordan algebra can be expressed as the sum of a nilpotent element and a scalar multiple of 1 , then the nilpotent elements of $J$ form an ideal. Also Tsai [253] in 1970 analyzed that an external characterization of the Levitzki radical of a Jordan ring $U$ as the intersection of a family of prime ideals $U$. He also discussed that by applying this characterization, it was easy to see that the Levitzki radical of a Jordan ring contains the prime radical of the same ring. For associative rings the same statement was well known, since the prime radical in associative rings was called the Baer radical. If the minimal condition on ideals holds on Jordan ring $U$, then the Levitzki radical, $L(U)$, and the prime radical, $R(U)$ of $U$ coincide. In 1971, McCrimmon [178] derived a general structure theory for noncommutative Jordan rings. He defined a Jacobson radical and showed it coincides with the nil radical for rings with descending chain condition on inner ideals; semisimple rings with D.C.C. were shown to be direct sums of simple rings, and the simple rings to be essentially the familiar ones. In addition he also obtained results, which seem to be new even in characteristic $\neq 2$, concerning algebras without finiteness conditions. He also showed that an arbitrary simple non-commutative Jordan ring containing two nonzero idempotent whose sum is not 1 is either commutative or quasi-associative. Erickson and Montgomery [46] in 1971 observed the special Jordan ring $R^{+}$, and when $R$ has an involution and $R$ is associative ring, the special Jordan ring $S$ of symmetric elements. They first showed that the prime radical of $R$ equals the prime radical of $R^{+}$, and that the prime radical of $R$ intersected with $S$ is the prime radical of $S$. Also they gave an elementary characterization, in terms of the associative structure of $R$, of primeness of $S$. Finally, they proved that a prime ideal of $R$ intersected with $S$ is a prime Jordan ideal of $S$. Also, in 1971, Shestakov [218] considered the class of non-commutative Jordan rings. This class generalized the class of rings introduced by Block [13] and Thedy [249]. Also he demonstrated, for rings of the given class, a theorem on nilpotency of null rings with a maximality condition for sub-rings and for anti-commutative rings satisfying the third Engel condition [152]. Moreover, he generalized nilpotency of finite-dimensional null algebras of the corresponding classes. Also shown that two sufficiently broad subclasses of the class of rings considered, there exists a locally nilpotent radical. He also considered finite-dimensional non-commutative Jordan algebra. In 1972, Lewand [160] examined some radical properties of quadratic Jordan algebras and showed that under certain conditions an ideal of a quadratic Jordan algebra is the radical.

In 1973, Britin [15] restricted his attention to the Jordan ring of symmetric elements of an 
associative ring with involution. Although he considered the problem of integral domains in this restricted case and his main result was more general. He used the approach via Goldie's theorem [113] for associative rings i.e.; $T$ has a ring of quotients which is semi-simple Artinian if and only if $T$ is semi-prime, contains no infinite direct sum of left ideals and satisfies A.C.C. on left annihilator ideals. He observed that if one replaced semi-prime by prime, then replaced semi-simple by simple. Then it can be shown that the conditions put on left ideals are implied by A.C.C. or D.C.C. on left ideals, when $T$ has an involution. In 1974, Britin [16] he obtained a Jordan ring of quotients for $H(R)$ by observing that if $R$ be a 2-torsion free semiprime associative ring with involution. Conditions are put on the Jordan ring $H(R)$ of symmetric elements which imply the existence of a ring of quotients which is a direct sum of involution simple artinian rings. Montgomery [184] in 1974 studied the concept of quotient rings in a special class of Jordan rings. It is worth mentioning that this concept was not developed in Jordan algebra before. In his work, he showed that if $R$ is an associative ring with involution and $J$ is a Jordan sub-ring of the symmetric elements containing the norms and traces of $R$, then if $J$ is a Jordan domain with the common multiple property, $J$ has a ring of quotients which is Jordan division algebra. Also, Ng Seong-Nam [211] in 1974 generalized the result of Osborn [192] which was basically proved for associative rings with involution. But Seong-Nam generalized the result for non-associative Jordan rings with involution. In addition, Loustao [161] in 1974 established some results regarding radical extensions of Jordan rings. Along the way, he proved analogies for Jordan rings of commutativity results for associative rings found in [90]. Further, he also extended commutativity results from $[49,127]$ to associative division algebras with involution whose symmetric elements are a radical extension of a commutative sub-algebra. In 1979, Petersson [197] completed the solution of the classification problem for locally compact Jordan division rings initiated in [196]. He also examined that a locally compact non-discrete Jordan division ring and a finite dimensional Jordan division algebra over that field. He also considered the centroid of a locally compact non-discrete field.

Moreover, in 1986, Slinko in his article [235] described the structure of a connected component of a locally compact alternative or Jordan ring. It was shown that each locally compact semiprime alternative or Jordan ring is a topological direct sum of its zero connected component, which is a semisimple finite-dimensional algebra over $R$ and a totally disconnected locally compact semiprime ring. This result can be viewed as a far reaching generalization of the classical Pontryagin theorem on connected associative locally compact skew fields. Furthermore, it was also proved that a connected locally compact alternative or Jordan ring having no nonzero idempotents is nilpotent and also established that the quasi-regular radical of an alternative or Jordan locally compact ring is closed. In 1986, Gonzalez et al., [56] introduced the order relation in Jordan rings, he proved that the relation $\leq$ defined by $x \leq y$ if and only if $x y=x^{2}, x^{2} y=x y^{2}=x^{3}$ is an order relation for a class of Jordan rings and proved that a Jordan ring $R$ is isomorphic to a direct product of Jordan division rings if and only if $\leq$ is a partial order on $R$ such that $R$ is hyperatomic and orthogonally complete. Later, in 1987, Garijo [54] discussed the Jordan regular ring associated with finite JBW- algebra. In this paper, he showed that every finite JBW-algebra $A$ is contained in a Von Neumann regular Jordan ring $A$ such that $A$ has no new idempotents. Moreover, he proved that every finite JBW- algebra has the common multiple property (non-associative analogous to the Ore condition) and that 
$a$ is the (unique) total ring of quotients of $A$. Hentzel and Peresi [84] in 1988 introduced almost Jordan rings. He proved that any Jordan ring with characteristic $\neq 2,3$ satisfies the identity: $2((a x) x) x+a((x x) x)=3(a(x x)) x$ along with commutativity implies the Jordan identity in any semiprime ring. In 1988, Slinko [236] generalized the result of Petersson [197] that any continuous Jordan division ring is finite-dimensional over its centroid. Secondly, he proved the condition of the solvability of the equations $x U a=b$, for $a \neq 0$. These conditions were actually required for the definition of Jordan division ring.

In 1993, Chuvakov [30] proved that in the class non-commutative Jordan rings satisfying the identity $([x, y], z, z)=0$ for an arbitrary radical $r$, any ideal of an $r$-semisimple ring is $r$-semisimple. Thus the problem of heredity of a radical $r$ in the class is equivalent to the problem of $r$-radicality of any ideal of an $r$-radical ring. He also proved that in the class of non-commutative Jordan rings $M$ a locally-nilpotent radical is hereditary.

For more and intrinsic study the readers are referred to the excellent books by Braun and Koecher [14] in 1966, Jacobson [114] in 1968 and McCrimmon [179] in 2004, on Jordan algebras which contain substantial material on general non-associative algebras. Also some relative research work can be found in the Proceedings of the international conferences on non-associative algebra and its applications [55, 156, 201].

In 2011, Radu [107] gave us an overview of the most important applications of Jordan structures inside mathematics and also to the physics. Nowadays, mathematics becomes more and more non-associative and the author predicts in his paper that in few years non-associativity will govern mathematics and applied sciences.

\subsection{Loop Rings (1944-2015)}

Historically, the concept of a non-associative loop ring according to our knowledge was first introduced in a paper by Bruck in 1944 [20]. Non-associative loop rings appeared to have been little more than a curiosity until the 1980s when the author found a class of non-associative Moufang loops whose loop rings satisfy the alternative laws. One can defined loop ring as given a loop $L$ and a commutative associative ring $R$ with 1 , one forms the loop ring $R L$ just as one would form a group ring if $L$ were a group. In the construction of $R L$ the binary operations addition " + " and multiplication "." are defined as follows $\alpha+\beta=\sum_{g \in L}\left(\alpha_{g}+\beta_{g}\right) g$ and $\alpha \beta=\sum_{g \in L}\left(\sum_{h k=g} \alpha_{h} \beta_{k}\right) g$.

In 1946, Bruck [22] revealed that the group ring result about the centre had a natural extension and he established a result regarding the centre of loop algebra, i.e.; the centre of loop algebra is spanned by conjugacy class sums. He also proved that a loop $R L$ is associative (commutative) if and only if $L$ is associative (commutative). In 1955, Paige [193] gave a striking example of phenomenon that the associative and commutative identities are very special, however in general, an identity in $L$ does not lift to $R L$ and an identity on $R L$ imposes much more than simply the same identity on $L$. He also proved that if $R$ is a ring of characteristic relatively prime to 30 and $L$ is a loop such that $R L$ is commutative and power associative, then $L$ is a group. In 1959, Hall [77] did an excellent work on right Moufang loops. A Moufang loop is a loop which satisfies this right Moufang identity: $((x y) z) y=x(y(z y))$. 
The Moufang identity is named for Ruth Moufang who discovered it in some geometrical investigations in the first half of this century [185]. Later, in 1974, Chein [25] discovered that any group is a Moufang loop, but here is a family of Moufang loops which are not associative.

In 1983, Goodaire [57] proved that if the Moufang identity on $L$ extends to a loop ring $R L$, then $R L$ must be an alternative ring. In 1985, Chein and Goodaire [26] presented the method of constructing all $R A$ loops, one which begins with the class of abelian groups possessing 2-torsion. They further determined when two $R A$ loops constructed by this method are isomorphic. In particular, they determined when two non-isomorphic groups with property $L C$ can both be embedded as index two sub-loops in the same $R A$ loop. Subsequently, in 1986, Goodair and Chein [27] worked with collaboration and yielded more satisfying information about $R A$ (right alternative) loops. Soon after, Goodaire and Parmenter [70] in 1986 demonstrated that the certain well known theorems concerning units in integral group rings holds more generally for integral loop rings which are alternative. Afterwards, in 1987, Goodaire and Parmenter [71] endeavored to establish conditions which guarantee the semi-simplicity of alternative loop rings with respect to any nil radical and with respect to the Jacobson radical. In 1988, Goodaire and Milies [63] first suggested to settle the isomorphism problem for alternative loop rings, it was shown that a Moufang loop whose integral loop ring is alternative is determined up to isomorphism by that loop ring. Secondly, it was shown that every normalized automorphism of an alternative loop ring $Z L$ is the product of an inner automorphism of $Q L$ and an automorphism of $L$. Additionally, in 1989, Goodaire and Milies [64] established that every torsion unit in an alternative loop ring over $Z$ is \pm a conjugate of a conjugate of a loop element. They also assumed that $Z L$ denotes the integral alternative loop ring of a finite loop $L$. It is a well-known result of Higman [95] that if $L$ is an abelian group then $\pm g, g \in L$ are the only torsion units (invertible elements of finite order) in $Z L$. When $L$ is not abelian, another obvious source of units is the set $\pm \gamma^{-1} g \gamma$ of conjugates of elements of $L$ by invertible elements in the rational loop algebra $Q L$. In the alternative but not associative case, one can form potentially more torsion units by considering conjugates of conjugates $\gamma_{1}^{-1}\left(\gamma_{2}^{-1} g \gamma_{2}\right) \gamma_{1}$ and so forth.

Furthermore, Chein and Goodaire [28] in 1990 continued their investigation of loops which gave rise to alternative loop rings. If the coefficient ring has characteristic2, these loops turn out to form a surprisingly wide class, in contrast to the situation of characteristic $\neq 2$. This paper described many properties of this class, includes diverse examples of Moufang loops which were united by the fact that they had loop rings which were alternative, and discussed analogues in loop theory of a number of important group theoretic constructions. In 1992, Vasantha Kandasamy [121] introduced a new notion in loop rings $K L$ called normal elements of the loop ring $K L$. An element $x \in K L$ is called a normal element of $K L$ if $\alpha K L=K L \alpha$. If every element of $K L$ is a normal element of $K L$ and called $K L$ the normal loop ring, also defined normal sub loop rings. Vasantha Kandasamy [122] in 1994 investigated a notion called strict right loop ring. He defined that if $L$ be a loop and $R$ a commutative ring with 1. The loop ring $R L$ is called the strict loop ring if the set of all ideals of $R L$ is ordered by inclusion. He also gave a class of loop rings, which were not strict loop rings. Moreover, Goodaire and Robinson [72] in 1994 exhibited a class of loops which have strongly right alternative loop rings that are not alternative. And they also proved fundamental propositions 
which generalized the necessary and sufficient conditions for a loop to have a strongly right alternative loop ring. Beside this in 1995, Vasantha Kandasamy [123] studied the mod $\mathrm{p}$ envelope of associative structure. He discussed the case of non-associative groups which were loops. That is in his study he replaced groups by loops. Again in 1995, Goodaire and Milies [65] further generalized and discussed few examples of Moufang loops whose loop rings are alternative, but not associative [57]. Since that time, there had been a great deal of work devoted to the study of such loops and to their loop rings. In their paper authors gave a brief discussion of those loops whose loop rings are alternative.

In 1996, Goodaire and Milies [66] considered an $R A$ loop is a loop whose loop rings, in characteristic different from 2, are alternative but not associative. Moreover, authors showed that every finite sub-loop $H$ of normalized units in the integral loop ring of an $R A$ loop $L$ is isomorphic to a sub-loop of $L$. They also showed that there exist units in the rational loop algebra. Thus, a conjecture of Zassenhaus which was open for group rings holds for alternative loop rings (which were not associative). In addition to this Goodaire and Robinson [73] in 1996 proposed the construction of loops $L$ which have right alternative loop rings $R L$ which were not left alternative. The construction generated loop rings $R L$ which are Bol and hence, right alternative merely set $z=1 e$ in the Bol identity $(x y . z) y=x(y z . y)$. Such loop rings are called strongly right alternative as they satisfied the more stringent condition. Barros and Juriaans [37] in 1996 discussed that Higman has proved a classical result giving necessary and sufficient conditions for the units of an integral group ring to be trivial. In this paper authors extended this result to a bigger class of diassociative loops which includes abelian groups, groups with a unique non-identity commutator, $R A$ loops, and other classes of loops. Again in 1997, Barros and Juriaans [38] proved the isomorphism problem for integral loop rings of finitely generated $R A$ loops using a decomposition of the loop of units. Also they described the finitely generated $R A$ loops whose loops of units satisfy a certain property. In 1998, Kunen [151] discussed that the right alternative law implies the left alternative law in loop rings of characteristic other than 2. He also exhibited a loop which failed to be right Bol loop, even though its characteristic 2 loop rings are right alternative. Also in 1999, Goodaire [58] sketched the brief history of loop rings which were not associative from early results of Bruck and Paige through the more recent discovery of alternative and right alternative rings and the work of Chein, Robinson and by the Goodaire.

In 2001, Bhandari and Kaila [11] observed that the additive as well as multiplicative Jordan decompositions hold in alternative loop algebras of finite $R A$ loops and the $R A$ loops for which the additive Jordan decomposition holds in the integral loop ring were characterized. Multiplicative Jordan decomposition (MJD) in $Z L$, where $L$ is a finite $R A$ loop with cyclic centre is analyzed, besides settling MJD for integral loop rings of all $R A$ loops of order $\leq 32$. It was also shown that for any finite $R A$ loop $L, \mu(Z L)$ is an almost splittable Moufang loop. Again in 2001, Goodaire and Milies [67] considered $L$ be an $R A$ loop, that is a loop whose loop ring in any characteristic is an alternative, but not associative ring. They also investigated necessary and sufficient conditions for the (Moufang) unit loop of $R L$ to be solvable when $R$ is the ring of rational integers or an arbitrary field. On the way Goodaire and Milies [68] in 2001 observed that an $R A$ loop has a torsion-free normal complement in the loop of normalized units of its integral loop ring. They also examined whether an $R A$ loop can be normal in its 
unit loop. Furthermore, in 2002, Nagy [187] showed that the fundamental ideal of loop ring $F L$ is nilpotent if and only if the multiplication group is $p$-group, where $p$ is prime, $L$ is finite loop of $p$-power order and $F$ is a field of characteristic $p$. Also in 2002, Vasantha Kandasamy and Parimala Kanthi [124] introduced a new class of Jordan loops of order $p+1$ where $p$ is a prime. They also proved this new class of Jordan loops is not Moufang loops or Bruck loops. Further they proved that the loop rings defined analogous to group rings by using a Jordan loop $J_{p}$ over rings which are commutative with unit or fields are Jordan rings only under special type of rings. Finally they showed that the loop ring in case of the Jordan loop $J_{p}$ over the ring $Z_{2}$ is a Jordan ring. Conversely if the loop ring $K L$ is a Jordan ring then the loop $L$ is a Jordan loop. Also explained that these new class of Jordan loops are power associative so the loop rings have proper associative sub-rings and these loop rings $K J_{p}$ had non-trivial zero divisors and idempotents. In 2005, Goodaire, Yuanlin Li and Parmenter [44] have considered $L$ as $R A$ loop, that is a loop whose loop rings are alternative, but not associative rings (in any characteristic). They investigated the necessary and sufficient conditions under which the hypercentral units in the integral loop ring $Z L$ are central. In 2006, Goodaire and Milies [69] discussed normality of $f$-unitary units in an alternative loop rings. In this paper, they also found necessary and sufficient conditions for $U_{f}(Z L)$ to be normal in $U(Z L)$ (the loop of all units in $Z L)$ where for $U_{f}(Z L)$ the set of all $f$-unitary units and $U(Z L)$ is the loop of all units in $Z L$.

Goodaire [61] in 2007 described some of the advances in the theory of loops whose loop rings satisfy interesting identities. He wrote this paper in memory of his friend Robinson with whom he did research. Again in 2007, Goodaire [62] discussed advances in the theory of loops whose loop rings satisfy interesting identities that had taken place primarily since 1998 . The major emphasis were on Bol loops that had strongly right alternative loop rings and on Jordan loops a hitherto largely ignored class of commutative loops some of whose loops rings satisfy the Jordan identity $\left(x^{2} y\right) x=x^{2}(y x)$. He raised a number of open questions and includes several suggestions for further research. Doostie and Pourfaraj [40] in 2007 studied the finite rings $Z_{p}[S]$ and $Z_{\left(p_{1} p_{2}\right)^{i}}\left[L_{n}(m)\right]$, and proved that the first one is commuting regular and the second ring contains the commuting regular element and idempotents as well (where $p, p_{1}$ and $p_{2}$ are odd primes. Moreover, $i, m$ and $n$ are positive integers such that $m<n,(m, n)=1$ and $(m-1, n)=1$. They also defined the commuting regular semigroup ring, commuting regular loop ring and commuting regular groupoid ring. In 2008, Chein et al., [188] established some connections between loops whose loop rings, in characteristic 2, satisfy the Moufang identities and loops whose loop rings, in characteristic 2, and satisfy the right Bol identities. Again in 2008, Chein and Goodaire [29] discussed that the possession of a unique non-identity commutator or associator was a property that dominates the theory of loops whose loop rings, while not associative, nevertheless satisfy an interesting identity. Furthermore, they also considered all loops with loop rings satisfying the right Bol identity (such loops are called SRAR) have been known to have this property. They presented various constructions of other kinds of SRAR loops. Also considered Bol loops whose left nucleus is an abelian group of index 2 and showed that the loop rings of some such loops were strongly right alternative and exhibited various SRAR loops with more than two commutators.

In 2009, Dart and Goodaire [36] investigated the existence of loop rings that were not 
associative but which satisfied the Moufang or Bol identities (without being associative). Their work turned out, with one exception, loop rings satisfying an identity of Bol-Moufang type all satisfy a Moufang or Bol identity. They also highlighted some similarities and differences in the consequences of several Bol- Moufang identities as they applied to loops and rings. Moreover, in 2012, Giraldo Vergara [254] discussed in details the developments of theory of loop rings that has been intrigued mathematicians from different areas. He also mentioned that in recent years, this theory has been developed largely, and as an example of this the complete description of the loop of invertible elements of the Zorn algebra is known to us. Recently, in 2014, Jayalakshmi and Manjula [118] investigated the case where the ring has characteristic 2 and extend to alternative loop rings by proving that the augmentation of order $2 n$ in characteristic 2 is a nilpotent ideal (of dimension $2 n-1$ ). This, of course, means that virtually all the familiar radicals of alternative rings coincide with the augmentation ideal. Also, in 2014, Jayalakshmi and Manjula [119] discussed that the right alternative law implies the left alternative law in loop rings of characteristic other than 2 . They also shown that there exists a loop which fails to be an extra loop, even though its characteristic 2 loop rings are right alternative.

\subsection{LA-Ring (2006-2016)}

After the concept of loop rings (1944), a new class of non-associative ring theory was given by Yusuf in 2006 [265]. Although the concept of LA-ring was given in 2006, but the systematic study and further developments was started in 2010 by Shah and Rehman in their paper [215]. It is worth mentioning that this new class of non-associative rings named Left almost rings (LA-ring) is introduced after a huge gap of 6 decades since the introduction of loop rings. Left almost rings (LA-ring) is actually an off shoot of LA-semigroup and LA-group. It is a noncommutative and non-associative structure and gradually due to its peculiar characteristics it has been emerging as useful non-associative class which intuitively would have reasonable contribution to enhance non-associative ring theory. By an LA- ring, we mean a non-empty set $R$ with at least two elements such that $(R,+)$ is an LA-group, $(R,$.$) is an LA-semigroup,$ both left and right distributive laws hold.

In [215], the authors have discussed LA-ring of finitely nonzero functions which is in fact a generalization of a commutative semigroup ring. They generalized the structure of commutative semigroup ring (ring of semigroup $S$ over ring $R$ represented as $R[X ; S]$ to a nonassociative LA-ring of commutative semigroup $S$ over LA-ring $R$ represented as $R\left[X^{s} ; s \in S\right]$, consisting of finitely nonzero functions. Nevertheless it also possesses associative ring structures. Furthermore they also discussed the LA-ring homomorphism. On the way the first ever definition of LA-module over an LA-ring was given by Shah and Rehman in the same paper [215].

Later in 2010, Shah et al., [217] introduced the notion of topological LA-groups and topological LA-rings which are some generalizations of topological groups and topological rings respectively. They extended some characterizations of topological groups and topological rings to topological LA-groups and topological LA-rings.

In 2011, Shah and Shah [213] established some basic and structural facts of LA-ring which 
will be useful for future research on LA-ring. They studied basic results such as if $R$ is an LA-ring then $R$ cannot be idempotent and also $(a+b)^{2}=(b+a)^{2}$ for all $a, b \in R$. If LA-ring $R$ has left identity $e$ then $e+e \neq e, e+0 \neq e$ and $e=(e+0)^{2}$. If $R$ is a cancellative LA-ring with left identity $e$ then $e+e=0$ and thus $a+a=0$ for all $a \in R$. An interesting result is that if $R$ is an LA-ring with left identity $e$ then right distributivity implies left distributivity. Also in 2011, Shah et al., [247] promoted the notion of LA-module over an LA-ring defined in [215] and further established the substructures, operations on substructures and quotient of an LA-module by its LA-sub module. They also indicated the non similarity of an LAmodule to the usual notion of a module over a commutative ring. Moreover, in 2011, Shah, Rehman and Raees [245] have generalized the concept of LA-ring by introducing the notion of near left almost ring (abbreviated as nLA-ring) $(R,+, \cdot) .(R,+)$ is an LA-group, $(R, \cdot)$ is an LA-semigroup and one distributive property of "." over " + " holds, where both the binary operations "+" and "." are non-associative. In continuation to [245], Shah, Ali and Rehman [246] in 2011 characterized nLA-ring through its ideals. They have shown that the sum of ideals is again an ideal, and established the necessary and sufficient condition for an nLA-ring to be direct sum of its ideals. Furthermore, they observed that the product of ideals is just a left ideal.

In 2012, Shah and Rehman [216] explored some notations of ideals and M-systems in LAring. They characterized LA-rings through some properties of their ideals. Moreover, they also established that if every subtractive subset of an LA-ring $R$ is semi-subtractive and also every quasi-prime ideal of an LA-ring $R$ with left identity $e$ is semi-subtractive. Also in 2012, Shah et al., [248] investigated the intuitionistic fuzzy normal sub-rings in non-associative rings. In their study they extended the notions for a class of non-associative rings i.e.; LA-ring. They established the notion of intuitionistic fuzzy normal LA-subrings of LA-rings. Specifically they proved that if an IFSA $=\left(\mu_{A}, \gamma_{A}\right)$ is an intuitionistic fuzzy normal LA-subring of an LA-ring $R$ if and only if the fuzzy sets $\mu_{A}$ and $\gamma_{A}$ are fuzzy normal LA-subrings of $R$. Also they showed that an IFSA $=\left(\mu_{A}, \gamma_{A}\right)$ is an intuitionistic fuzzy normal LA-subring of an LA-ring $R$ if and only if the fuzzy sets $\mu_{A}$ and $\gamma_{A}$ are anti-fuzzy normal LA-subrings of $R$.

In 2013, a notable development was done by Rehman et al., [106] when the existence of LA-ring was shown by giving the non-trivial examples of LA-ring. The authors showed the existence of LA-ring using the mathematical program Mace4. With the existence of nontrivial LA-ring, ultimately the authors were able to abolish the ambiguity about the associative multiplication because the first example on LA-ring given by Yusuf [265] was trivial. Also in 2013, Gaketem [53] studied the properties of quasi-ideals of $P$-regular nLA-ring which is in fact a generalization of LA-ring.

In 2014, Alghamdi and Sahraoui [8] broaden the concept of LA-module given in the paper [215] by constructing a tensor product of LA-modules. Although, LA-groups and LA-modules need not to be abelian, the new construction behaves like standard definition of the tensor product of usual modules over a ring. They also then extended some simple results from the ordinary tensor to the new setting. In addition, Yiarayong [263] in 2014 studied left ideals, left primary and weakly left primary ideals in LA-rings. Some characterizations of left primary and weakly left primary ideals were obtained. Moreover, the author investigated relationships 
of left primary and weakly left primary ideals in LA-rings. Finally, he obtained necessary and sufficient conditions of a weakly left primary ideal to be a left primary ideal in LA-rings.

Recently, in 2015, Hussain and W. Khan [104] characterized LA-rings by congruence relations. They had shown that each homomorphism of LA-rings defines a congruence relation on LA-rings. They also then discussed quotient LA- rings. At the end they proved analogue of the isomorphism theorems for LA-rings. Also Shah and Asima Razzaque in their paper [214] discussed soft non-associative rings and explore some of its algebraic properties. The notions of soft M-systems, soft P-systems, soft I-systems, soft quasi-prime ideals, soft quasi-semiprime ideals, soft irreducible and soft strongly irreducible ideals were introduced and several related properties were investigated. Moreover in 2016, Shah et al., [244] taken a step forward to apply the concepts of soft set theory to LA-ring by introducing soft LA-rings, soft ideals, soft prime ideals, idealistic soft LA-rings and soft LA-homomorphism. They provided a number of examples to illustrate these concepts.

\section{Conclusions}

Nowadays, mathematics is becoming more and more non-associative and it is a general prediction that in few years' non-associativity will govern mathematics and applied sciences. We would like to point out that application of non-associative ring theory is astonishing and has become an instrumental in parts of physics, quantum mechanics, atomic spectroscopy, solid state physics, differential and algebraic geometry, differential equations, space time theory and etc. In this paper we tried to present the complete survey of all types of non-associative rings and enumerate some of their various applications and developments in different directions to date. We do believe that this survey would be unique in its own way for the reason that such comprehensive and complete information regarding all types of non-associative rings under one umbrella can hardly be found. We hope that this work will provide an endless source of inspiration for future research in non-associative ring theory.

\section{References}

[1] I. D. Ado. On representations of finite continuous groups using linear substitutions. Izvestiya Kazanskogo Fiziko-Matematicheskogo Obshchestva, 7:1-43, 1934-35.

[2] A. A. Albert. Quadratic forms permitting composition. Ann. of Math., 43:161-177, 1942 .

[3] A. A. Albert. Quasigroups I. Trans. Amer. Math. Soc., 54:507-519, 1943.

[4] A. A. Albert. Quasigroups II. Trans. Amer. Math. Soc., 55:401-409, 1944.

[5] A. A. Albert. Power associative rings. Trans. Amer. Math. Soc., 64:552-593, 1948.

[6] A. A. Albert. On the right alternative algebras. Ann. of Math., 50:318-328, 1949.

[7] A. A. Albert. On simple alternative rings. Canad. J. Math., 4:129-135, 1952. 
[8] A. M. Alghamdi and F. Sahraoui. Tensor product of LA-modules. International Mathematical Forum, 9:1309-1319, 2014.

[9] J. C. Baez. The Octonions. Bull. Amer. Math. Soc., 39:145-205, 2002.

[10] J. G. F. Belinfante and B. Kolman. A survey of Lie groups and Lie algebras with applications and computational methods. SIAM J. Appl. Math., Society for Industrial and Applied Mathematics Philadelphia, 1989.

[11] A. K. Bhandari and A. Kaila. Jordan decompositions in alternative loop rings. Rend. Circ. Mat. Palermo, 50(2), 2001.

[12] G. Birkhoff. Representability of Lie algebras and Lie groups by matrices. Ann. of Math., 38:526-532, 1937.

[13] R. E. Block. A unification of the theories of Jordan and alternative algebras. Notices Amer. Math. Soc., 16:389-412, 1969.

[14] H. Braun and M. Koecher. Jordan-Algebren [German]. Springer-Verlag, Berlin-New York, 1966.

[15] D. J. Britten. On prime Jordan rings H(R) with chain condition. J. Algebra, 27:414421, 1973.

[16] D. J. Britten. On semiprime Jordan rings $\mathrm{H}(\mathrm{R})$ with A.C.C. Proc. Amer. Math. Soc., 45:175-178, 1974.

[17] B. Brown and N. H. McCoy. Some theorems on groups with applications to ring theory. Trans. Amer. Math. Soc., 69:302-311, 1950.

[18] B. Brown and N. H. McCoy. Prime ideals in non-associative rings. Trans. Amer. Math. Soc., 89:245-255, 1958.

[19] R. B. Brown. A new type of non-associative algebras. Proc. Natl. Acad. Sci. USA, 50:947-948, 1963.

[20] R. H. Bruck. Some results in the theory of linear non-associative algebras. Trans. Amer. Math. Soc., 56:141-199, 1944.

[21] R. H. Bruck. Some results in the theory of quasigroups. Trans. Amer. Math. Soc., $56: 19-52,1944$.

[22] R. H. Bruck. Contributions to the theory of loops. Trans. Amer. Math. Soc., 60:245$354,1946$.

[23] R. H. Bruck. A survey of binary systems. Springer-Verlag, Berlin, Heidelberg, 1958.

[24] R. H. Bruck and E. Kleinfeld. The structure of alternative division rings. Proc. Amer. Math. Soc., 2:878-890, 1951. 
[25] O. Chein. Moufang loops of small order I. Trans. Amer. Math. Soc., 188:31-51, 1974.

[26] O. Chein and E. G. Goodaire. Isomorphism of loops which have alternative loop rings. Comm. Algebra, 13:1-20, 1985.

[27] O. Chein and E. G. Goodaire. Loops whose loop rings are alternative. Comm. Algebra, 14:293-310, 1986.

[28] O. Chein and E. G. Goodaire. Loops whose loop rings in characteristic 2 are alternative. Comm. Algebra, 18:659-688, 1990.

[29] O. Chein and E. G. Goodaire. SRAR loops with more than two commutators. $J$. Algebra, 319:1903-1912, 2008.

[30] V. P. Chuvakov. Heredity of radicals in a class of non-commutative Jordan rings. Math. Notes, 54:1267-1273, 1993.

[31] P. Coelho and U. Nunes. Lie algebra application to mobile robot control: a tutorial. Robotica, 21:483-493, 2003.

[32] P. M. Cohn. A non-nilpotent Lie ring satisfying the Engel condition and a nonnilpotent Engel group. Math. Proc. Cambridge Philos. Soc., 51:401-405, 1955.

[33] J. H. Conway and D. A. Smith. On quaternions and octonions. A. K. Peters, Massachusetts, 2003.

[34] J. F. Cornwell. Group Theory in Physics: An Introduction. Academic Press, San Diego, 1997.

[35] D. Eswara Rao D. Bharathi and P. Ravi. Right nucleus in right alternative rings. International Journal of Mathematical Archive, 4:252-255, 2013.

[36] B. C. Dart and E. G. Goodaire. Loop rings satisfying identities of bol- moufang type. J. Algebra Appl, 8:401-411, 2009.

[37] Luiz G. X. de Barros and S. O. Juriaans. Units in Integral loop rings. J. Algebra, 183:637-648, 1996.

[38] Luiz G. X. de Barros and S. O. Juriaans. Units in alternative integral loop rings. Results Math., 31:266-281, 1997.

[39] G. M. Dixon. Division algebras: Octonions, quaternions, complex numbers and the algebraic design of physics. Springer-Science, Brandeis University, USA, 1994.

[40] H. Doostie and L. Pourfaraj. Finite rings and loop rings involving the commuting regular elements. International Mathematical Forum, 2:2579-2586, 2007. 
[41] G. V. Dorofeev. One example in the theory of alternative rings. Sibirsk. Mat. Zh., 4:1049-1052, 1963.

[42] R. Dubisch and S. Perlis. On the radical of a non-associative algebra. Amer. J. Math., 70:540-546, 1948.

[43] E. B. Dynkin. Computation of the coefficients in the Campbell-Hausdorff formula. Dokl. Akad. Nauk SSSR, 57:323-326, 1947.

[44] Yuanlin Li E. G. Goodaire and M. M. Parmenter. Hypercentral units in alternative loop rings. J. Algebra, 283:317-326, 2005.

[45] N. Yu. Makarenko E. I. Khukhro and P. Shumyatsky. Frobenius groups of automorphisms and their fixed points. Forum Math., 26:73-112, 2011.

[46] T. S. Erockson and S. Montgomery. The prime radical in special Jordan rings. Trans. Amer. Math. Soc., 156:155-164, 1971.

[47] H. Essannouni and A. Kaidi. Semiprime alternative rings with A.C.C. Lecture Notes in Math., 1328:82-93, 1988.

[48] H. Essannounia and A. Kaidi. Goldie's theorem for alternative rings. Proc. Amer. Math. Soc., 121:39-45, 1994.

[49] C. Faith. Radical extensions of rings. Proc. Amer. Math. Soc., 12:274-283, 1961.

[50] B. L. M. Ferreira and R. Nascimento. Derivable maps on alternative rings. Rev. Cinc. Exatas Nat., 16:9-15, 2014.

[51] A. Forsythe and N. H. McCoy. On the commutativity of certain rings. Bull. Amer. Math. Soc., 52:523-526, 1946.

[52] A. T. Gainov. Identical relations for binary-Lie rings. Uspekhi Mat. Nauk, 3:141-146, 1957.

[53] T. Gaketem. Quasi-ideals of a p-regular near left almost rings. International Journal of Pure and Applied Mathematics, 87:219-227, 2013.

[54] P. J. Garijo. The Jordan regular ring associated to a finite JBW-algebra. J. Algebra, 110:56-73, 1987.

[55] S. Gonzalez. Non-associative algebra and its applications. Kluwer, Dordrecht, Proc. of the Third Intl. Conf. Oviedo, Spain, 1994.

[56] S. Gonzalez and C. Martinez. Order relation in Jordan rings and a structural theorem. Proc. Amer. Math. Soc., 98:379-388, 1986.

[57] E. G. Goodaire. Alternative loop rings. Publ. Math. Debrecen, 30:31-38, 1983. 
[58] E. G. Goodaire. A brief history of loop rings. Mat. Contemp., 16:93-109, 1999.

[59] E. G. Goodaire. Nilpotent right alternative rings and Bol circle loops. Comm. Algebra, 28:2445-2459, 2000.

[60] E. G. Goodaire. Units in right alternative loop rings. Publ. Math. Debrecen, 59:353$362,2001$.

[61] E. G. Goodaire. Advances in loop rings and their loops. Quasigroups Related Systems, 15:1-18, 2007.

[62] E. G. Goodaire. More on the History of loop rings. 2007.

[63] E. G. Goodaire and C. P. Milies. Isomorphisms of integral alternative loop rings. Rend. Circ. Mat. Palermo, 37(2):126-135, 1988.

[64] E. G. Goodaire and C. P. Milies. Torsion units in alternative loop rings. Proc. Amer. Math. Soc., 107:7-15, 1989.

[65] E. G. Goodaire and C. P. Milies. Ring alternative loops and their loop rings. Resenhas do Instituto de Matemtica e Estatstica da Universidade de So Paulo, 2:47-82, 1995.

[66] E. G. Goodaire and C. P. Milies. Finite subloops of units in an alternative loop ring. Proc. Amer. Math. Soc., 124:995-1002, 1996.

[67] E. G. Goodaire and C. P. Milies. Alternative loop rings with solvable unit loops. J. Algebra, 240:25-39, 2001.

[68] E. G. Goodaire and C. P. Milies. Normal subloops in the integral loop ring of an RA loop. Canad. Math. Bull., 44:27-35, 2001.

[69] E. G. Goodaire and C. P. Milies. Normality of f-unitary units in an alternative loop rings. J. Algebra Appl., 5:537-548, 2006.

[70] E. G. Goodaire and M. M. Parmenter. Units in alternative loop rings. Israel J. Math., 53:209-216, 1986.

[71] E. G. Goodaire and M. M. Parmenter. Semi-simplicity of alternative loop rings. Acta Math. Hungar., 50:241-247, 1987.

[72] E. G. Goodaire and D. A. Robinson. A Class of loops with right alternative loop rings. Comm. Algebra, 22:5623-5634, 1994.

[73] E. G. Goodaire and D. A. Robinson. A Construction of loops which admit right alternative loop rings. Results Math., 29:56-62, 1996.

[74] F. Grsey and C. H. Tze. On the role of division, Jordan and related algebras in particle physics. World Scientific, Singapore, 1996. 
[75] M. Hall. A basis for free Lie rings and higher commutators in free groups. Proc. Amer. Math. Soc., 1:575-581, 1950.

[76] M. Hall and Jr. An identity in Jordan rings. Proc. Amer. Math. Soc., 7:990-998, 1956.

[77] M. Hall and Jr. The theory of groups. MacMillan, New York, 1959.

[78] Harish-Chandra. Faithful representations of lie algebras. Ann. of Math., 50:68-76, 1949.

[79] B. Hartley and T. Meixner. Finite soluble groups containing an element of prime order whose centralizer is small. Arch. Math. (Basel), 36:211-213, 1981.

[80] H. Hashimoto. On -Modular right ideals on an alternative ring. Journal of the Faculty of Science, Hokkaido University, 15(Ser. 1, Mathematics):131-133, 1960.

[81] F. Hausdorff. Die symbolische exponentialformel in der gruppentheorie. Ber Verh Saechs Akad Wiss Leipzig, 58:19-48, 1906.

[82] I. R. Hentzel. Right alternative rings with idempotents. J. Algebra, 17:303-309, 1971.

[83] I. R. Hentzel. Generalized right alternative rings. Pacific J. Math., 60:95-102, 1975.

[84] I. R. Hentzel and L. A. Peresi. Almost Jordan rings. Proc. Amer. Math. Soc., 104:343-348, 1988.

[85] I. N. Herstein. On the Lie and Jordan rings of a simple associative ring. Amer. J. Math., 77:279-285, 1955.

[86] I. N. Herstein. The Lie ring of a simple associative ring. Duke Math. J., 22:471-476, 1955.

[87] I. N. Herstein. Lie and Jordan systems in simple rings with involution. Amer. J. Math., 78:629-649, 1956.

[88] I. N. Herstein. Jordan derivatives of prime rings. Proc. Amer. Math. Soc., 8:11041110, 1957.

[89] I. N. Herstein. Lie and Jordan structures in simple associative rings. Bull. Amer. Math. Soc., 67:517-531, 1961.

[90] I. N. Herstein. Non-commutative rings. The Carus Mathematical Monographs15, MAA Textb, 1968.

[91] I. N. Herstein. Topics in Ring Theory. University of Chicago Press, Chicago, 1969. 
[92] I. N. Herstein. On the Lie structure of an associative ring. J. Algebra, 14:561-571, 1970.

[93] I. N. Herstein and E. Kleinfeld. Lie mappings in characteristic 2. Pacific J. Math., 10:843-852, 1960.

[94] P. J. Higgins. Lie rings satisfying the engel condition. Math. Proc. Cambridge Philos. Soc., 50:8-15, 1954.

[95] G. Higman. The units of group rings. Proc. Lond. Math. Soc., 46:231-248, 1940.

[96] G. Higman. Groups and rings having automorphisms without non-trivial fixed elements. J. Lond. Math. Soc., 32:321-332, 1957.

[97] C. Hopkins. Rings with minimal condition for left ideals. Ann. of Math., 40:712-730, 1939.

[98] M. Horn and S. Zandi. Second cohomology of Lie rings and the schur multiplier. Int. J. Group Theory, 3:9-20, 2014.

[99] R. Howe. Very basic Lie theory. Amer. Math. Monthly, 90:600-623, 1983.

[100] M. M. Humm. On a class of right alternative rings without nilpotent ideals. $J$. Algebra, 5:164-174, 1967.

[101] M. M. Humm and E. Kleinfeld. On free alternative rings. Journal of Combinatorial Theory, 2:140-144, 1967.

[102] J. E. Humphreys. Introduction to Lie algebras and representation theory. Springer, New York, 1972.

[103] J. E. Humphreys and E. James. Introduction to Lie algebras and representation theory. Springer, New York, 1994.

[104] F. Hussain and W. Khan. Congruences on left almost rings. International Journal of Algebra and Statistics, 4:1-6, 2015.

[105] F. Klein I. M. Yaglom and S. Lie. Evolution of the idea of symmetry in the nineteenth century. Birkhauser, Boston, 1988.

[106] T. Shah I. Rehman, M. Shah and Asima Razzaque. On existence of non-associative LA-rings. Stiint. Univ. "Ovidius" Constanta Ser. Mat., 21:223-228, 2013.

[107] Radu Iordanescu. Jordan structures in mathematics and physics. arXiv preprint arXiv:1106.4415, 2011.

[108] K. Iwasawa. On the representation of Lie algebras. Jpn. J. Math., 19:405-426, 1948. 
[109] B. Kolman J. G. Belinfante and H. A. Smith. An introduction to Lie groups and Lie algebras with applications. SIAM Rev., 8:11-46, 1966.

[110] N. Jacobson. Cayley numbers and normal simple Lie algebras of type G. Duke Math. J., 5:775-783, 1939.

[111] N. Jacobson. Isomorphisms of Jordan rings. Amer. J. Math., 70:317-326, 1948.

[112] N. Jacobson. The centre of a Jordan ring. Bull. Amer. Math. Soc., 54:316-322, 1948.

[113] N. Jacobson. Structure of rings. American Mathematical Society Transl. Ser. 2, USA, 1964.

[114] N. Jacobson. Structure and representations of Jordan algebra. Amer. Math. Soc. Colloq. Publ., USA, 1968.

[115] N. Jacobson and C. E. Rickart. Jordan homomorphisms of rings. Trans. Amer. Math. Soc., 60:479-502, 1950.

[116] N. Jacobson and C. E. Rickart. Homomorphisms of Jordan rings of self-adjoint elements. Trans. Amer. Math. Soc., 72:310-322, 1952.

[117] K. Jayalakshmi and S. Madhavi Latha. Right nucleus in generalized right alternative rings. International Journal of Research-Granthaalayah, 3:1-12, 2015.

[118] K. Jayalakshmi and C. Manjula. Bol loops in nilpotent alternative loop ring. International Journal of Statistika and Mathematika, 10:56-60, 2014.

[119] K. Jayalakshmi and C. Manjula. Extra and alternative loop rings. International Journal of Scientific and Research Publications, 4, 2014.

[120] C. R. Jordan and D. A. Jordan. Lie rings of derivations of associative rings. J. Lond. Math. Soc., 2:33-41, 1978.

[121] W. B. V. Kandasamy. On normal elements in loop rings. Ultra Scien. of Phys. Sci., 4:210-212, 1992.

[122] W. B. V. Kandasamy. On strictly right loop rings. J. of Harbin Inst. of Sci. and Tech., 18:116-118, 1994.

[123] W. B. V. Kandasamy. A note on the modular loop ring of a finite loop. Opuscula Math., 15:109-112, 1995.

[124] W. B. V. Kandasamy and J. M. Parimala Kanthi. Loop rings of jordan rings. National symposium on mathematical methods and applications, Indian Institute of Technology, Madras, Chennai, TN, India, 2002.

[125] I. Kaplansky. Topological rings. Amer. J. Math., 69:153-183, 1947. 
[126] I. Kaplansky. Topological rings. Bull. Amer. Math. Soc., 54:809-826, 1948.

[127] I. Kaplansky. A theorem on division rings. Canad. J. Math., 3:290-292, 1951.

[128] N. Kawamoto. On prime ideals of Lie algebras. Hiroshima Math. J., 4:679-684, 1974.

[129] E. I. Khukhro. Finite p-groups admitting an automorphism of order p with a small number of fixed points. Math. Notes, 38:867-870, 1986.

[130] E. I. Khukhro. Lie rings and Lie Groups admitting an almost regular automorphism of prime order. Sb. Math., 71:51-63, 1992.

[131] E. I. Khukhro and N. Yu. Makarenko. Lie rings with almost regular automorphisms. J. Algebra, 264:641-664, 2003.

[132] E. I. Khukhro and N. Yu. Makarenko. Finite groups and Lie rings with a metacyclic frobenius group of automorphisms. J. Algebra, 386:77-104, 2013.

[133] E. Kleineelo. Right alternative rings. Proc. Amer. Math. Soc., 4:939-944, 1953.

[134] E. Kleinfeld. Right alternative rings. Proc. Amer. Math. Soc., 4(6):939-944, 1953.

[135] E. Kleinfeld. Simple alternative rings. Ann. of Math., 58:544-547, 1953.

[136] E. Kleinfeld. Generalization of a theorem on simple alternative rings. Port. Math., 14:91-94, 1955.

[137] E. Kleinfeld. A characterization of the cayley numbers. Studies in Modern Algebra, MAA Studies in Mathematics, 2:126-143, 1963.

[138] E. Kleinfeld. Middle nucleus-center in a simple Jordan ring. J. Algebra, 1:40-42, 1964.

[139] E. Kleinfeld. On right alternative rings without proper right ideals. Pacific J. Math., 31:87-102, 1969.

[140] E. Kleinfeld. Generalization of alternative rings II. J. Algebra, 18:326-339, 1971.

[141] E. Kleinfeld. On a generalization of Alternative and Lie rings. Trans. Amer. Math. Soc., 155:385-395, 1971.

[142] E. Kleinfeld. Anti-commutative elements in alternative rings. J. Algebra, 83:65-71, 1983.

[143] E. Kleinfeld and H. F. Smith. On prime right alternative rings with commutators in the left nucleus. Bull. Aust. Math. Soc., 49:287-298, 1994.

[144] L. A. Kokoris. Some nodal non-commutative Jordan algebras. Proc. Amer. Math. Soc., 9:164-166, 1958. 
[145] A. I. Kostrikin. Lie rings satisfying the Engel condition. Izvestiya Akademii Nauk USSR, 21:515-540, 1957.

[146] A. I. Kostrikin. On the relation between periodic groups and Lie rings. Izvestiya Akademii Nauk USSR, 21:289-310, 1957.

[147] A. I. Kostrikin. On the burnside problem. Dokl. Akad. Nauk SSSR, 119:1081-1084, 1958.

[148] V. A. Kreknin. The solubility of Lie algebras with regular automorphisms of finite period. Dokl. Math., 4:683-685, 1963.

[149] V. A. Kreknin. Solvability of a Lie algebra containing a regular automorphism. Sib. Math. J., 8:536-537, 1967.

[150] V. A. Kreknin and A.I. Kostrikin. Lie algebras with regular automorphisms. Dokl. Math., 4:355-358, 1963.

[151] K. Kunen. Alternative loop rings. Comm. Algebra, 26:557-564, 1998.

[152] E. N. Kuzmin. On anti-commutative algebras satisfying the Engel condition. Sibirsk. Mat. Zh., 8:1026-1034, 1967.

[153] F. Kuzucuoglu. Isomorphisms of the unitriangular groups and associated Lie rings for the exceptional dimensions. Acta Appl. Math., 85:209-213, 2005.

[154] M. G. Lozano L. A. Crdenas and J. R. Calvio. The maximal left quotient rings of alternative rings. Comm. Algebra, 33:1031-1042, 2005.

[155] M. G. Lozano L. A. Crdenas and J. R. Calvio. On quotient rings in alternative rings. Comm. Algebra, 42:5464-5473, 2014.

[156] L. SbitnevaI L. Sabinin and P. Shestakov. Non-associative algebra and its applications. Taylor Francis / CRC Press, New York, 2005.

[157] C. Lanski and S. Montgomery. Lie structure of prime rings of characteristic 2. Pacific J. Math., 42:117-136, 1972.

[158] M. Lazard. Sur les algebres enveloppantes universelles des certaines algebres de Lie. Publications Scientifiques de l'Universite d'Alger, 2(A1):281-294, 1954.

[159] M. Lazard. Sur les groupes nilpotents et les anneaux de Lie. Ann. Sci. Ec. Norm. Super., 71:101-190, 1954.

[160] R. Lewand. Hereditary radicals in Jordan rings. Proc. Amer. Math. Soc., 33:302-306, 1972.

[161] J. A. Loustao. Radical extensions of Jordan rings. J. Algebra, 30:1-11, 1974. 
[162] M. G. Lozano and M. S. Molina. Left quotient rings of alternative rings. J. Algebra Appl., 6:71-102, 2007.

[163] K. Kunen M. K. Kinyon and J. D. Phillips. Strongly right alternative rings and Bol loops. arXiv preprint math/0508005, 2005.

[164] I. Macdonald. On certain varieties of groups. Math. Z., 76:270-282, 1961.

[165] I. Macdonald. On certain varieties of groups II. Math. Z., 78:175-188, 1962.

[166] W. Magnus. Uber Beziehungen zwischen hoheren Kommutatoren. Journal fur die reine und angewandte Mathematik, 117:105-115, 1937.

[167] N. Yu. Makarenko. Finite 2-groups with automorphisms of order 4. Algebra Logic, 40:47-54, 2001.

[168] N. Yu. Makarenko. A nilpotent ideal in the Lie rings with automorphisms of prime order. Sib. Math. J., 46:1097-1107, 2005.

[169] N. Yu. Makarenko and E. I. Khukhro. Nilpotent groups admitting an almost regular automorphism of order four. Algebra Logic, 35:176-187, 1996.

[170] N. Yu. Makarenko and E. I. Khukhro. On Lie rings admitting an automorphism of order 4 with few fixed points. Algebra Logic, 35:21-43, 1996.

[171] N. Yu. Makarenko and E. I. Khukhro. Lie rings admitting automorphisms of order 4 with few fixed points II. Algebra Logic, 37:78-91, 1998.

[172] N. Yu. Makarenko and E. I. Khukhro. Almost solvability of Lie algebras with almost regular automorphisms. Dokl. Math., 68:325-326, 2003.

[173] N. Yu. Makarenko and E. I. Khukhro. Almost solubility of Lie algebras with almost regular automorphisms. J. Algebra, 227:370-407, 2004.

[174] A. I. Malcev. On a representation of non-associative rings. Uspekhi Mat. Nauk, 1:181-185, 1952 .

[175] A. I. Malcev. Analytic loops. Mat. Sb., 78:569-576, 1955.

[176] K. McCrimmon. A general theory of Jordan rings. Proc. Natl. Acad. Sci. USA, 56:1072-1079, 1966.

[177] K. McCrimmon. Nondegenerate Jordan rings are von Neumann regular. J. Algebra, 11:111-115, 1969.

[178] K. McCrimmon. Non-commutative Jordan rings. Trans. Amer. Math. Soc., 158:133, 1971.

[179] K. McCrimmon. A Taste of Jordan Algebras. Springer-Verlag, New York, 2004. 
[180] Y. A. Medvedev. Groups and Lie algebras with almost regular automorphisms. $J$. Algebra, 164:877-885, 1994.

[181] Yu. Medvedev. p-Divided Lie rings and p-groups. J. Lond. Math. Soc., 59:787-798, 1999.

[182] K. Meyberg. The fundamental-formula in Jordan rings. Arch. Math., 21:43-44, 1970.

[183] I. M. Miheev. On prime right alternative rings. Algebra Logika, 14:56-60, 1975.

[184] S. Montgomery. Rings of quotients for a class of special Jordan rings. J. Algebra, 31:154-165, 1974.

[185] R. Moufang. Alternativkrper und der Satz vom vollstndigen Vierseit (D9). Abh. Math. Semin. Univ. Hambg., 9:207-222, 1933.

[186] R. Moufang. Zur Struktur von Alternative pern. Math. Ann., 110:416-430, 1935.

[187] G. P. Nagy. On nilpotent loop rings and a problem of Goodaire. Publ. Math. Debrecen, 61:549-554, 2002.

[188] E. G. Goodaire O. Chein and M. Kinyon. When is a right alternative loop ring that is also right Bol actually Moufang. arXiv preprint arXiv:0803.2205, 2008.

[189] J. J. O'Connor and E. F. Robertson. Maruis Sophus Lie. The Mac Tutor History of Mathematics Archive, 2000.

[190] S. Okubo. Introduction to octonion and other non-associative algebras in physics. Cambridge University Press, Cambridge, 1995.

[191] J. M. Osborn. Jordan and associative rings with nilpotent and invertible elements. J. Algebra, 15:301-308, 1970.

[192] J. M. Osborn. Varieties of algebras. Adv. Math., 8:163-369, 1972.

[193] L. J. Paige. A theorem on commutative power associative loop algebras. Proc. Amer. Math. Soc., 6:279-280, 1955.

[194] A. C. Paul and Md. Sabur Uddin. Lie and Jordan structure in simple gamma rings. J. Phys. Sci., 14:77-86, 2010.

[195] A. C. Paul and Md. Sabur Uddin. Lie structure in simple gamma rings. Int. J. Pure Appl. Sci. Technol., 4:63-70, 2010.

[196] H. P. Petersson. Lokal kompakte Jordan-divisions ringe. Abh. Math. Semin. Univ. Hambg., 39:164-179, 1973.

[197] H. P. Petersson. Classification of locally compact Jordan division rings. J. Algebra, 58:350-360, 1979. 
[198] H. P. Petersson. Composition algebras over algebraic curves of genus zero. Trans. Amer. Math. Soc., 337:473-491, 1993.

[199] H. O. Pflugfelder. Quasigroups and loops: Introduction. Heldermann Verlag, Berlin, 1990.

[200] H. O. Pflugfelder. Historical notes on loop theory. Comment. Math. Univ. Carolin., 41:359-370, 2000.

[201] H. Guzzo Jr R. Costa, A. Grishkov and L. A. Peresi. Non-associative Algebra and its Applications. CRC Press, Marcel Dekker, New York, 2000.

[202] I. Rehman. On generalized commutative rings and related structures. PhD thesis, Quaid-i-Azam University, Islamabad, Pakistan, 2011.

[203] M. Rich. The Prime radical in alternative rings. Proc. Amer.Math. Soc., 56:11-15, 1976.

[204] B. I. Rose. Model theory of alternative rings. Notre Dame J. Form. Log., 19:215-243, 1978.

[205] R. D. Schafer. Alternative algebras over an arbitrary field. Bull. Amer. Math. Soc., 49:549-555, 1943.

[206] R. D. Schafer. Inner derivations of non-associative algebras. Bull. Amer. Math. Soc., 55:769-776, 1949.

[207] R. D. Schafer. The Wedderburn principal theorem for alternative algebras. Bull. Amer. Math. Soc., 55:604-614, 1949.

[208] R. D. Schafer. Non-commutative Jordan algebras of characteristic zero. Proc. Amer. Math. Soc., 6:472-475, 1955.

[209] R. D. Schafer. On non-commutative Jordan algebras. Proc. Amer. Math. Soc., 9:110-117, 1958.

[210] R. D. Schafer. Restricted non-commutative Jordan algebras of characteristic p. Proc. Amer. Math. Soc., 9:141-144, 1958.

[211] Ng Seong-Nam. Jordan rings with involution. Trans. Amer. Math. Soc., 200:111139, 1974.

[212] Ng Seong-Nam. Right nucleus in Right alternative algebras. J. Lond. Math. Soc., 21(2):456-464, 1980.

[213] M. Shah and T. Shah. Some basic properties of LA-rings. International Mathematical Forum, 6:2195-2199, 2011. 
[214] T. Shah and Asima Razzaque. Soft M-systems in a class of soft non-associative rings. U.P.B. Sci. Bull., Series A, 77(3):131-142, 2015.

[215] T. Shah and I. Rehman. On LA-rings of finitely non-zero functions. Int. J. Contemp. Math. Sciences, 5:209-222, 2010.

[216] T. Shah and I. Rehman. On characterizations of LA-rings through some properties of their ideals. Southeast Asian Bull. Math., 36:695-705, 2012.

[217] T. Shah and K. Yousaf. Topological LA-groups and LA-rings. Quasigroups Related Systems, 18:95-104, 2010.

[218] I. P. Shestakov. Certain classes of non-commuative Jordan rings. Algebra Logic, 10:252-280, 1971.

[219] A. I. Shirshov. On the representation of Lie rings in associative rings. Uspekhi Mat. Nauk, 5:173-175, 1953.

[220] A. I. Shirshov. Some problems in the theory of rings that are nearly associative. Uspekhi Mat. Nauk, 13:3-20, 1958.

[221] L. A. Skorniakov. Alternative division rings. Ukrain. Mat. Zh., 2:70-85, 1950.

[222] L. A. Skorniakov. Alternative division rings of characteristic 2 and 3. Uspekhi Mat. Nauk, 2:94-99, 1950.

[223] L. A. Skorniakov. Projective planes. Ukrain. Mat. Zh., 6:112-154, 1951.

[224] L. A. Skorniakov. Projective planes. Ukrain. Mat. Zh., 15:177-184, 1951.

[225] L. A. Skorniakov. Right alternative fields. Izvestia Akad. Nauk SSSR Ser. Math., 15:177-184, 1951.

[226] M. Slater. Weakly prime alternative rings (abstract). Notices Amer. Math. Soc., 12:367-368, 1965.

[227] M. Slater. Nucleus and center in alternative rings. J. Algebra, 7:372-388, 1967.

[228] M. Slater. Ideals in semiprime alternative rings. J. Algebra, 8:60-76, 1968.

[229] M. Slater. Alternative rings with D.C.C.I. J. Algebra, 11:102-110, 1969.

[230] M. Slater. Alternative rings with D.C.C. II. J. Algebra, 14:464-484, 1970.

[231] M. Slater. Prime alternative rings I. J. Algebra, 15:229-243, 1970.

[232] M. Slater. Prime alternative rings II. J. Algebra, 15:244-251, 1970.

[233] M. Slater. The socle of an alternative ring. J. Algebra, 14:443-463, 1970. 
[234] M. Slater. Alternative rings with D.C.C. III. J. Algebra, 18:179-200, 1971.

[235] A. M. Slinko. Locally compact alternative and Jordan rings. Algebra Logic, 25:274$278,1986$.

[236] A. M. Slinko. Locally compact Jordan rings that are nearly division rings. Math. Notes, 43:409-415, 1988.

[237] M. F. Smiley. Alternative regular rings without nilpotent elements. Bull. Amer. Math. Soc., 53:775-778, 1947.

[238] M. F. Smiley. The radical of an alternative ring. Ann. of Math., 49:702-709, 1948.

[239] M. F. Smiley. Jordan homomorphisms and right alternative rings. Proc. Amer. Math. Soc., 8:668-671, 1957.

[240] M. F. Smiley. Kleinfeld's proof of the Bruck-Kleinfeld-Skornyakov theorem. Math. Ann., 134:53-57, 1957.

[241] R. L. San Soucie. Right alternative division rings of characteristic 2. Proc. Amer. Math. Soc., 6:291-296, 1955.

[242] R. L. San Soucie. Right alternative rings of characteristic two. Proc. Amer. Math. Soc., 6:716-719, 1955.

[243] S. Suanmali. On the relationship between the class of a Lie algebra and the classes of its subalgebras. International Journal of Algebra and Computation, 18:83-95, 2008.

[244] Asima Razzaque T. Shah and I. Rehman. Application of Soft Sets to Non-Associative Rings. Journal of Intelligent and Fuzzy Systems, 30(3):1537-1546, 2016.

[245] F. Rehman T. Shah and M. Raees. On near left almost rings. International Mathematical Forum, 6:1103-1111, 2011.

[246] G. Ali T. Shah and F. Rehman. Direct sum of ideals in a generalized LA-ring. International Mathematical Forum, 6:1095-1101, 2011.

[247] M. Raees T. Shah and G. Ali. On LA-modules. Int. J. Contemp. Math. Sciences, 6:999-1006, 2011.

[248] N. Kausar T. Shah and I. Rehman. Intuitionistics fuzzy normal subring over a non-associative ring. An. Stiint. Univ. "Ovidius" Constanta Ser. Mat., 20:369-386, 2012 .

[249] A. Thedy. Concerning the wedderburn factorization theorem. Math. Z., 113:173-195, 1970.

[250] A. Thedy. Right alternative rings. J. Algebra, 37:1-43, 1975. 
[251] C. E. Tsai. The Prime radical in Jordan rings. Proc. Amer. Math. Soc., 19:1171$1175,1968$.

[252] C. E. Tsai. A Characterization of the maximal von-neumann regular ideal in Jordan rings. J. Algebra, 12:227-230, 1969.

[253] C. E. Tsai. The Levitzki radical in Jordan rings. Proc. Amer. Math. Soc., 24:119$123,1970$.

[254] C. R. Giraldo Vergara. A walk through the loop rings. Rev. Integr. Temas Mat., $30: 15-24,2012$.

[255] J. P. Ward. Quaternions and cayley numbers. Kluwer Academic Publishers, Dordrecht, Boston, 1997.

[256] G. P. Wene. Alternative rings whose symmetric elements are or a right multiple is symmetric idempotent. Pacific J. Math., 90:483-492, 1980.

[257] A. Widiger. Alternative rings in which every proper right ideal is maximal. Fund. Math., 116:165-167, 1983.

[258] James B. Wilson. More characteristic subgroups, Lie rings and isomorphism tests for p-groups. J. Group Theory, 16:875-897, 2013.

[259] James B. Wilson. New Lie products for groups and their automorphisms. arXiv preprint arXiv:1501.04670, 2015.

[260] E. Witt. Treue Darstellung Liescher Ringe. Journal fur die reine und angewandte Mathematik, 177:152-160, 1937.

[261] E. Witt. Treue Darstellung beliebiger Liescher Ringe. Collect. Math., 6:107-114, 1953.

[262] E. Witt. Die Unterringe der freien Liescher Ringe. Math. Z., 64:195-216, 1956.

[263] P. Yiarayong. On left primary and weakly left primary ideals in LA-rings. Asian Journal of Applied Sciences, 2(4):457-463, 2014.

[264] Dr. A. Anjaneyulu Y.s.n.Satyanarayana and Dr. D. prabhakara Reddy. Peculiarity of nucleus in alternative rings. International Journal of Engineering Research and General Science, 3:556-561, 2015.

[265] S. M. Yusuf. On left almost rings. In Proc. of 7th International Pure Math., Islamabad, Pakistan, 2006. Pakistan Mathematical Society, Quaid-i-Azam University.

[266] A. J. Zapirain. On almost regular automorphisms of finite p-groups. Adv. Math., 153:391-402, 2000. 
[267] A. I. Zhukov. Complete systems of defining relations in non-associative algebras. Mat. Sb., 69:267-280, 1950.

[268] M. Zorn. Theorie der alternativen ringe. Abh. Math. Semin. Univ. Hambg., 8:123$147,1930$.

[269] M. Zorn. Alternativkr per und quadratische Systme. Abh. Math. Semin. Univ. Hambg., 9:395-402, 1933.

[270] M. Zorn. The automorphisms of Cayley's non-associative algebra. Proc. Natl. Acad. Sci. USA, 21:355-358, 1935.

[271] M. Zorn. Alternative rings and related questions I: Existence of the radical. Ann. of Math., 42:676-686, 1941. 